QUARTERLY OF APPLIED MATHEMATICS

VOLUME LXIII, NUMBER 4

DECEMBER 2005, PAGES 747-778

S $0033-569 X(05) 00981-7$

Article electronically published on October 6, 2005

\title{
T-CONVERGENCE FOR A FAULT MODEL WITH SLIP-WEAKENING FRICTION AND PERIODIC BARRIERS
}

\author{
BY \\ IOAN R. IONESCU (Laboratoire de Mathématiques, Université de Savoie, 73376 Le Bourget-du-Lac \\ Cedex, France), \\ DANIEL ONOFREI (Department of Mathematical Sciences, Worcester Polytechnic Institute, \\ Worcester, Massachusetts 01609), \\ AND \\ BOGDAN VERNESCU (Department of Mathematical Sciences, Worcester Polytechnic Institute, \\ Worcester, Massachusetts 01609)
}

Abstract. We consider a three-dimensional elastic body with a plane fault under a slip-weakening friction. The fault has $\epsilon$-periodically distributed holes, called (smallscale) barriers. This problem arises in the modeling of the earthquake nucleation on a large-scale fault.

In each $\epsilon$-square of the $\epsilon$-lattice on the fault plane, the friction contact is considered outside an open set $T_{\epsilon}$ (small-scale barrier) of size $r_{\epsilon}<\epsilon$, compactly inclosed in the $\epsilon$-square. The solution of each $\epsilon$-problem is found as local minima for an energy with both bulk and surface terms. The first eigenvalue of a symmetric and compact operator $K^{\epsilon}$ provides information about the stability of the solution.

Using $\Gamma$-convergence techniques, we study the asymptotic behavior as $\epsilon$ tends to 0 for the friction contact problem. Depending on the values of $c=: \lim _{\epsilon \rightarrow 0} r_{\epsilon} / \epsilon^{2}$ we obtain different limit problems.

The asymptotic analysis for the associated spectral problem is performed using $G$ convergence for the sequence of operators $K^{\epsilon}$. The limits of the eigenvalue sequences and the associated eigenvectors are eigenvalues and respectively eigenvectors of a limit operator.

From the physical point of view our result can be interpreted as follows:

i) if the barriers are too large (i.e. $c=\infty$ ), then the fault is locked (no slip),

Received March 11, 2005.

2000 Mathematics Subject Classification. Primary 35J25, 74Q05; Secondary 35P99, 74B10.

Key words and phrases. $\Gamma$-convergence, slip-weakening friction, Steklov problem.

E-mail address: ionescu@univ-savoie.fr

E-mail address: onofrei@wpi.edu

E-mail address: vernescu@wpi.edu 
ii) if $c>0$, then the fault behaves as a fault under a slip-dependent friction. The slip weakening rate of the equivalent fault is smaller than the undisturbed fault. Since the limit slip-weakening rate may be negative, a slip-hardening effect can also be expected.

iii) if the barriers are too small (i.e. $c=0$ ), then the presence of the barriers does not affect the friction law on the limit fault.

1. Introduction. The origin of friction has been found in the hard contacts between two rough surfaces, and the the geometry of the contact, let us say the roughness, has been shown to be a decisive parameter for frictional behavior 33. Since friction is a phenomenon that concerns both microscopic and macroscopic scales, the contact on a geological fault is also modelled at the scale of the seismic waves (i.e. kilometric).

The friction properties are likely heterogeneous on the fault, particularly with the presence of barriers. By the term barrier, we denote a patch on the fault plane where no slip occurs. This concept cannot be applied for the evolution of the fault at the geological time scale, but it has been shown to be useful and relevant in the description of fault heterogeneity during an earthquake [30, 31].

The macroscopic behavior of a fault with small-scale heterogeneity of rupture resistance (small-scale barriers) is difficult to relate to the local properties of the fault. A formal measure of the friction on the fault itself would just be a local particular law, that is, varying with the position along the fault. In this paper we focus on the following question: How can we obtain an effective (equivalent) friction law which, used on a homogeneous fault, leads to a slip evolution similar to the one produced on the heterogeneous fault?

Mathematically the problem is related to the homogenization of the Newmann Sieve problem for the Laplacian studied by several authors [15, 14, 5, 12]. In the geophysical context the problem was studied (see [11, 10, 32]) in two dimensions (anti-plane geometry) to obtain the rescaling of the weakening rate through a spectral analysis.

The Newmann Sieve problem associated to the linear elasticity operator was studied by Lobo and Perez [25, 8. An extension to the non-linear case of the Neumann Sieve has been studied by Ansini in 2. Our friction problem is similar to the previous one, with the important difference being that the tangential component of the displacement has zero jump on the barriers, and the limit analysis is therefore developed on a larger functional space.

Let us outline the contents of the paper.

In section 2 we consider the three-dimensional shearing of an elastic domain which contains an internal boundary (the fault) located on a plane (the fault plane). The contact on the fault is described through a slip-weakening friction (i.e. the decrease of the friction force with slip). This friction law is used in the geophysical context of earthquake modeling, and experimental studies 29. pointed out the good agreement of this model with experimental data. The symmetry of the displacement field with respect to the fault plane (see for instance 20] for the geophysical meaning) gives an important simplification of the problem: the normal over-stress on the fault vanishes. The fact that the normal stress has a weak variation of during the dynamic rupture was already observed in direct computations [4, 26] as well as in the inversion of seismological data 
[13. An important consequence of the above assumption is the fact that we can associate to the physical problem a minimization problem for the energy function. In modelling seismic phenomena, where at least two equilibria (before and after an earthquake) are involved, the energy function cannot be supposed convex.

In section 3 we obtain (as in [24] under slightly different assumptions) sufficient conditions of stability through the first eigenvalue of the tangent problem. Since this eigenvalue problem has an important significance in the description of the physical properties of the fault, we shall study it in the next section.

In section 4 we give the main results of the paper. First we set the perturbed (or heterogeneous) problem: a fault which has $\epsilon$-periodically distributed barriers of radius $r_{\epsilon}$. For $0<c=: \lim _{\epsilon \rightarrow 0} r_{\epsilon} / \epsilon^{2}<\infty$ we prove that the sequence of energy functionals $\Gamma$ converges to a limit energy functional. For the proof of liminf and the limsup inequalities we adapt an idea from [2].

The limit functional is associated to another slip-weakening friction problem called the equivalent friction law. In the last part of this section we prove that the eigenvalues and eigenfunctions of the perturbed tangent problem converge to the eigenvalues and eigenfunctions of the equivalent (limit) tangent problem. For this we adapt G-convergence techniques, developed for the Newmann-Sieve problem in [27].

The slip-weakening rate of the equivalent (or limit) fault is smaller than the undisturbed fault. Since the limit slip-weakening rate may be negative, a slip-hardening effect can also be expected. Moreover, we have to point out that even if the small-scale friction law is isotropic, the equivalent one is not. This surprising fact is natural if we have in mind that the periodic distribution of the barriers is not isotropic, hence the limit problem will inherit this anisotropic geometrical perturbation. We have to mention here that this property was also obtained [8, 25] for an elastic body with a surface having small no-slip regions. We make the observation that the proof of the convergence is based on the explicit computation of the solution for the cell problem, which in our case cannot be easily computed because of the general mixed-type boundary conditions on the parts of the boundary. This is the reason we chose the $\Gamma$-convergence approach in our paper.

In the last section we give the physical interpretation of the previous theoretical results in the context of a barrier erosion process during the earthquake nucleation (or initiation) phase, which precedes the dynamic rupture. We point out the important role played by the process of erosion of the barriers in the effective properties of the homogenized fault. We deduce from our analysis that the nucleation phase can be divided into three time periods. First we are dealing with a locking stage with no "macroscopic" slip. The second time period is characterized by a smaller, and even negative, weakening rate and by the loss of the isotropy of the friction law. The third time period corresponds to the last stage of (effective) initiation when the friction properties are the same with the undisturbed fault.

2. Statement of the physical problem. We consider the three-dimensional shearing of an elastic domain $\mathcal{D} \subset \mathbb{R}^{3}$. If we denote by $u: \mathcal{D} \longrightarrow \mathbb{R}^{3}$ the displacement field, 


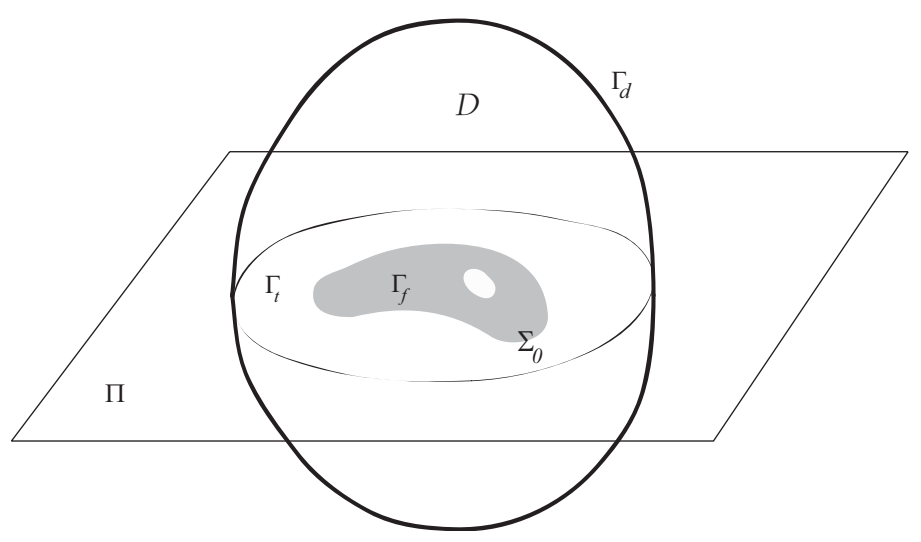

FIG. 1. The geometry of the 3-D problem

then the elastic constitutive equation and the equilibrium equation read

$$
\sigma(u)=\mathcal{A} \epsilon(u), \quad \operatorname{div}(\mathcal{A} \epsilon(u))=0 \quad \text { in } \mathcal{D},
$$

where $\mathcal{A}$ is the fourth-order elastic tensor, $\sigma(u)$ is the over stress tensor and $\epsilon(u)=$ $\frac{1}{2}\left(\nabla u+\nabla^{T} u\right)$ is the small strain tensor. $\mathcal{A}$ is a symmetric and positively defined fourthorder tensor, i.e.

$$
\mathcal{A}_{i j k l} \in L^{\infty}(\mathcal{D}), \quad \mathcal{A}(x) \epsilon \cdot \sigma=\mathcal{A}(x) \sigma \cdot \epsilon, \quad \text { a.e. } \quad x \in \mathcal{D},
$$

such that $\mathcal{A}(x) \epsilon \cdot \epsilon \geq M_{1}|\epsilon|^{2}$ and $|\mathcal{A}(x) \epsilon| \leq M_{2}|\epsilon|$ a.e $x \in \mathcal{D}$ with $M_{1}, M_{2}>0$,

for all $i, j, k, l=\overline{1,3}$ and for all $\sigma, \epsilon \in \mathbf{R}_{S}^{3 \times 3}$.

The smooth boundary $\Sigma=\partial \mathcal{D}$ is divided into two disjoint parts, $\Sigma=\Sigma_{d} \cup \Gamma_{f}$ : $\Sigma_{d}=\partial \overline{\mathcal{D}}$ the exterior boundary and $\Gamma_{f}$ the interior one (i.e. it is a subset of the interior of $\overline{\mathcal{D}}$ ). For the sake of simplicity on the exterior boundary we shall suppose vanishing displacement conditions, i.e. $u=0$ on $\Sigma_{d}$. The interior boundary is located in the plane $\Pi=\left\{x_{3}=0\right\}$, and will be called the fault or fault region. We assume that the pre-stress $\sigma^{\infty} \in C^{0}(\overline{\mathcal{D}})$ is such that the fault does not open. Moreover the fault $\Gamma_{f}$ is under a slip-dependent friction law:

$$
\begin{gathered}
{\left[\sigma_{i 3}(u)\right]=0, \quad i=\overline{1,3}, \quad\left[u_{3}\right]=0, \quad \text { on } \Gamma_{f},} \\
\sigma_{\tau}(u)+\tau^{\infty}=-\mu\left(\left|\left[u_{\tau}\right]\right|\right)\left|\sigma_{33}(u)-S\right| \frac{\left[u_{\tau}\right]}{\left|\left[u_{\tau}\right]\right|} \quad \text { if } \quad\left[u_{\tau}\right] \neq 0 \text { on } \Gamma_{f}, \\
\left|\sigma_{\tau}(u)+\tau^{\infty}\right| \leq \mu(0)\left|\sigma_{33}(u)-S\right| \quad \text { if }\left[u_{\tau}\right]=0 \text { on } \Gamma_{f},
\end{gathered}
$$

where [ ] denotes half of the jump across $\Gamma_{f}$ (i.e. $\left.[w]=\left(w^{+}-w^{-}\right) / 2\right), \sigma_{\tau}(u)=$ $-\left(\sigma_{13}(u), \sigma_{23}(u), 0\right)$ is the tangential over-stress, $\sigma_{33}(u)$ is the normal over-stress, $u_{\tau}=$ $\left(u_{1}, u_{2}, 0\right)$ is the tangential displacement, and $\tau^{\infty}=:-\left(\sigma_{13}^{\infty}, \sigma_{23}^{\infty}, 0\right)$ and $-S=: \sigma_{33}^{\infty}$ are the tangential and the normal pre-stress acting on $\Gamma_{f}$. From the above assumptions on $\sigma^{\infty}$ we have $S, \tau_{i}^{\infty} \in C^{0}\left(\Gamma_{f}\right)$. Equations (2.5)-(2.6) assert that the tangential (friction) stress is bounded by the normal stress multiplied by the value of the friction coefficient $\mu(0)$. If this limit is not attained, sliding does not occur. Otherwise the friction stress is opposed 
to the slip $\left[u_{\tau}\right]$, and its absolute value depends on the slip modulus through $\mu\left(\left|\left[u_{\tau}\right]\right|\right)$. Concerning the regularity of $\mu: \mathbb{R}_{+} \rightarrow \mathbb{R}_{+}$we suppose that the friction coefficient is a Lipschitz function with respect to the slip, i.e. there exists $L_{\mu} \geq 0$ such that

$$
\left|\mu\left(s_{1}\right)-\mu\left(s_{2}\right)\right| \leq L_{\mu}\left|s_{1}-s_{2}\right|,
$$

and we denote by $H$ its antiderivative

$$
H(u):=\int_{0}^{u} \mu(s) d s .
$$

We suppose that there exists $\gamma \in L^{\infty}\left(\mathbb{R}_{+}\right)$and $a \geq 0$ such that

$$
H(r)-H(s) \geq \mu(s)(r-s)-\gamma(s)(r-s)^{2} / 2-a|r-s|^{3}, \quad \forall r, s \geq 0 .
$$

Let us remark that if $\mu$ is two times differentiable with a bounded second derivative, then (2.8) holds with $\gamma(s)=-\mu^{\prime}(s)$. If $\mu$ is continuous but only piecewise differentiable, then (2.8) holds with $\gamma(s)=-\min \left\{\mu^{\prime}(s+), \mu^{\prime}(s-)\right\}$.

A specific friction law with a linear piecewise slip weakening, which is a reasonable approximation of the experimental observations (see [29]), can be written as follows:

$$
\mu(s)= \begin{cases}\frac{\mu_{d}-\mu_{s}}{D_{c}} s+\mu_{s} & \text { if } s \leq D_{c} \\ \mu_{d} & \text { if } s \geq D_{c}\end{cases}
$$

where $\mu_{s}>\mu_{d}$ are the static and, respectively, dynamic friction coefficients, and $D_{c}$ is the critical slip. In this case $\gamma(x)=\left(\mu_{s}-\mu_{d}\right) / D_{c}$.

In the following we shall suppose that $\mathcal{D}$ is symmetric with respect to the plane $\Pi$. As in 20] the following symmetries of the displacement field with respect to the plane $\Pi$ will be considered:

$$
u_{1}\left(x,-x_{3}\right)=-u_{1}\left(x, x_{3}\right), \quad u_{2}\left(x,-x_{3}\right)=-u_{2}\left(x, x_{3}\right), \quad u_{3}\left(x,-x_{3}\right)=u_{3}\left(x, x_{3}\right),
$$

where $x=\left(x_{1}, x_{2}\right)$ and $(x, 0)$ belongs to $\Sigma_{0}$, the intersection of $\overline{\mathcal{D}}$ with the plane $\Pi$. In the case of an isotropic elastic material, i.e.

$$
\mathcal{A}_{i j k l}=\lambda \delta_{i j} \delta_{k l}+G \delta_{i k} \delta_{j l},
$$

with $\lambda, G>0$ the Lamé coefficients, we deduce the following symmetries of the stress field $\sigma_{33}$ :

$$
\sigma_{33}\left(x,-x_{3}\right)=-\sigma_{33}\left(x, x_{3}\right) .
$$

The condition of continuity of the stress vector (2.4) on the fault plane $\Gamma_{f}$ gives the fact that the normal over-stress $\sigma_{33}$ does not present any variation during the slip

$$
\sigma_{33}\left(x, 0^{+}\right)=\sigma_{33}\left(x, 0^{-}\right)=0, \quad \text { for any }(x, 0) \in \Sigma_{0} .
$$

Since the displacement field is continuous outside the faults, from the symmetry conditions (2.10) we get that the tangential displacement is vanishing outside $\Gamma_{f}$ :

$$
u_{1}\left(x, 0^{+}\right)=u_{1}\left(x, 0^{-}\right)=u_{2}\left(x, 0^{+}\right)=u_{2}\left(x, 0^{-}\right)=0, \quad \text { for all }(x, 0) \in \Sigma_{0} \backslash \Gamma_{f},
$$

and the jump on $\Gamma_{f}$ is given by

$$
\left[u_{i}(x, 0)\right]=u_{i}\left(x, 0^{+}\right)=-u_{i}\left(x, 0^{-}\right), \quad i=1,2, \quad \text { for all }(x, 0) \in \Gamma_{f} .
$$


Let us denote by $\Omega:=\mathcal{D} \cap\left\{x_{3}>0\right\}$ the upper half of the domain $\mathcal{D}$ and by $\Gamma_{d}:=$ $\Sigma_{d} \cap\left\{x_{3}>0\right\}, \quad \Gamma_{t}:=\Sigma_{0} \backslash \Gamma_{f}$, which implies that $\partial \Omega=\Gamma_{d} \cup \Gamma_{t} \cup \Gamma_{f}$. From the above symmetry properties we can restrict ourselves to the upper half $\Omega$ of $\mathcal{D}$. We state the problem $(\mathcal{P})$ : find the displacement field $u: \Omega \rightarrow \mathbb{R}^{3}$ solution of

$$
\begin{gathered}
\sigma(u)=\mathcal{A} \epsilon(u), \quad \operatorname{div}(\mathcal{A} \epsilon(u))=0 \text { in } \Omega, \\
u=0 \text { on } \Gamma_{d}, \quad \sigma_{33}(u)=0, u_{\tau}=0 \text { on } \Gamma_{t}, \\
\sigma_{33}(u)=0, \quad\left\{\begin{array}{l}
\sigma_{\tau}(u)=-S \mu\left(\left|u_{\tau}\right|\right) \frac{u_{\tau}}{\left|u_{\tau}\right|}-\tau^{\infty} \text { if } u_{\tau} \neq 0, \\
\left|\sigma_{\tau}(u)+\tau^{\infty}\right| \leq S \mu(0) \quad \text { if } u_{\tau}=,
\end{array} \text { on } \Gamma_{f} .\right.
\end{gathered}
$$

3. Existence and stability. Let us denote by $V$ the closed subspace of $\left[H^{1}(\Omega)\right]^{3}$ given by

$$
V:=\left\{v \in\left[H^{1}(\Omega)\right]^{3} / v=0 \quad \text { on } \quad \Gamma_{d}, \quad v_{\tau}=0 \text { on } \Gamma_{t}\right\} .
$$

From Korn's inequality and Poincare's inequality one can easily deduce that the inner product

$$
\langle u, v\rangle_{V}:=\int_{\Omega} \mathcal{A} \epsilon(u) \cdot \epsilon(v), \quad \forall u, v \in V,
$$

generates a norm, denoted by \|\|$_{V}$, which is equivalent with the natural norm on $\left[H^{1}(\Omega)\right]^{3}$ and

$$
M_{1}\|D u\|_{L^{2}}^{2} \leq\|u\|_{V}^{2} \leq M_{2}\|D u\|_{L^{2}}^{2}, \quad \forall u \in V
$$

We have the following variational formulation of the physical problem (2.15)-(2.17) (see also [24]):

$$
u \in V, \quad\langle u, u-v\rangle_{V}+j(u, u)-j(u, v) \leq f(u-v), \quad \forall v \in V,
$$

where $j: V \times V \longrightarrow \mathbf{R}_{+}$and $f: V \longrightarrow \mathbf{R}$ are given by

$$
j(u, v)=\int_{\Sigma_{0}} S \mu\left(\left|u_{\tau}\right|\right)\left|v_{\tau}\right|, \quad f(v)=-\int_{\Sigma_{0}} \tau^{\infty} \cdot v_{\tau} \quad \forall u, v \in V .
$$

Let us now introduce the total energy functional $\mathcal{W}: V \longrightarrow \mathbf{R}$ given by

$$
\mathcal{W}(v)=\frac{1}{2}\|v\|_{V}^{2}+\int_{\Sigma_{0}} S H\left(\left|v_{\tau}\right|\right)-f(v), \quad \forall v \in V,
$$

which characterizes the "physically acceptable" solutions. Indeed we have the following result.

Theorem 3.1. If $u \in V$ is a local minimum for $\mathcal{W}$, then $u$ is a solution of (3.3). Moreover there exists a global minimum for $\mathcal{W}$, i.e. there exists $u \in V$ such that

$$
\mathcal{W}(u) \leq \mathcal{W}(v), \quad \forall v \in V .
$$

Proof. Let $u$ be a local minimum, i.e. there exists $\delta$ such that $\mathcal{W}(u) \leq \mathcal{W}(w)$ for all $w \in V$ with $\|w-u\|_{V} \leq \delta$. For all $v \in V$ we put $w=u+t(v-u)$, with $t>0$ small enough, in the last inequality and we pass to the limit with $t \rightarrow 0$ to deduce (3.3).

In order to prove that $\mathcal{W}$ has a global minimum we remark that the trace map is compact from $V$ to $L^{2}\left(\Gamma_{f}\right)$. Hence $v \rightarrow \int_{\Gamma_{f}} S H\left(\left|v_{\tau}\right|\right)-f(v)$ is weakly continuous on $V$, which implies that $\mathcal{W}$ is weakly lower semicontinuous. Bearing in mind that $\lim \inf \mathcal{W}(v)=\infty$ 
for $\|v\|_{V} \rightarrow \infty$, from a Weierstrass-type theorem we deduce that $\mathcal{W}$ has at least one global minimum.

Let us now consider the following eigenvalue problem, which will be useful to characterize the stability of the local minima, $(\mathcal{E})$ : find $u: \Omega \longrightarrow \mathbb{R}^{3}, u \neq 0$ and $\lambda \in \mathbf{R}$ such that

$$
\begin{gathered}
\sigma(u)=\mathcal{A} \epsilon(u), \quad \operatorname{div} \sigma(u)=0, \text { in } \Omega, \\
u=0 \quad \text { on } \Gamma_{d}, \quad \sigma_{33}(u)=0, \quad u_{\tau}=0 \quad \text { on } \Gamma_{t}, \\
\sigma_{33}(u)=0, \quad \sigma_{\tau}(u)=\lambda u_{\tau} \quad \text { on } \Gamma_{f},
\end{gathered}
$$

which has the following variational formulation:

$$
u \in V, \quad\langle u, v\rangle_{V}=\lambda \int_{\Gamma_{f}} u_{\tau} \cdot v_{\tau}, \quad \forall v \in V .
$$

The same technique as in 24] can be used to get the structure of the spectrum. For the convenience of the reader we shall give the proof.

Theorem 3.2. The eigenvalues and eigenfunctions of (3.10) form a sequence $\left(\lambda_{n}, u_{n}\right)_{n \geq 1}$ with $0<\lambda_{1} \leq \lambda_{2} \leq \ldots$ and $\lambda_{n} \longrightarrow+\infty$. Moreover we have

$$
\frac{\left\|u_{1}\right\|_{V}^{2}}{\int_{\Gamma_{f}}\left|u_{1 \tau}\right|^{2} d x}=\lambda_{1}=\min _{v \in V} \frac{\|v\|_{V}^{2}}{\int_{\Gamma_{f}}\left|v_{\tau}\right|^{2} d x}
$$

Proof. Let $L=\left\{f=\left(f_{1}, f_{2}, 0\right) / f_{1}, f_{2} \in L^{2}\left(\Sigma_{0}\right), f_{1}=f_{2}=0\right.$ on $\left.\Gamma_{t}\right\}$ be a closed subspace of $\left[L^{2}\left(\Sigma_{0}\right)\right]^{3}$. Denote by $\gamma_{\tau}: V \rightarrow L$ the compact operator which associates to all $v \in V$ the tangential component of its trace on $\Gamma_{f}$, i.e.

$$
\gamma_{\tau}(v) \doteq v_{\tau}=v-(v \cdot n) n \quad \text { along } \Gamma_{f}
$$

for any $v \in V$.

Let $V_{1}=\operatorname{ker} \gamma_{\tau}$. Using the definition of $V$ we can see that

$$
V_{1}=\left\{v \in V / v_{\tau}=0 \quad \text { on } \Sigma_{0}\right\} .
$$

Now consider

$$
W=V_{1}^{\perp}=\left\{v \in V /\langle v, w\rangle_{V}=0 \quad \forall w \in V_{1}\right\} .
$$

Let $P_{W}: V \rightarrow W$ be the orthogonal projection onto $W$ and define $T: L \rightarrow W$ to be the linear and bounded operator which associates to each $f \in L$ the unique solution $T(f) \in W$ of the following linear equation:

$$
\langle T(f), v\rangle_{V}=\int_{\Gamma_{f}} f \cdot v_{\tau} d x, \quad \forall v \in V .
$$

We can now define the linear bounded operator $K: W \rightarrow W$ by $K v \doteq T\left(v_{\tau}\right)$. From (3.12) we get

$$
\langle K u, v\rangle_{V}=\int_{\Gamma_{f}} u_{\tau} v_{\tau}
$$

for all $u, v \in W$, which implies that $K$ is symmetric compact and strictly positive. Hence $K$ has a positive and decreasing sequence of eigenvalues $\left(\beta_{n}\right)_{n \geq 1}$ with $\beta_{n} \rightarrow 0$ and an orthonormal sequence of corresponding eigenvectors, $\left(u_{n}\right)_{n \geq 1}$. 
It is easy to observe that $\lambda_{n} \doteq \frac{1}{\beta_{n}}$ will be the eigenvalues of the problem $(\mathcal{E})$ and $u_{n}$ will be the orthonormal eigenvectors corresponding to it. Then Rayleigh's principle for $K$ gives us the statement of the theorem.

The following theorem makes use of the first eigenvalue of the above spectral problem to give sufficient conditions for a solution of (3.3) to be stable.

TheOREM 3.3. Let $u \in V$ be a solution of $\left(\underline{3.3)}\right.$ and let $\lambda_{1}$ be the first eigenvalue of $\mathcal{E}$. If

$$
\lambda_{1}>c_{u}=: \text { ess } \sup _{x \in \Gamma_{f}} S(x) \gamma\left(\left|u_{\tau}(x)\right|\right),
$$

where $\gamma$ has been defined in (2.8) and $-S$ is the normal stress on $\Gamma_{f}$, then $u$ is an isolated local minimum for $\mathcal{W}$, i.e. there exists $\delta>0$ such that

$$
\mathcal{W}(u)<\mathcal{W}(v) \quad \forall v \in V, v \neq u,\|v-u\|_{V}<\delta .
$$

Proof. Let us suppose that $u$ is not a local minimum for $\mathcal{W}$, i.e. there exists $\left(v_{m}\right)_{m} \subset$ $V, v_{m} \rightarrow u$ and $\mathcal{W}\left(v_{m}\right) \leq \mathcal{W}(u)$. If we put $v=v_{m}$ in (3.3) from the last inequality and from (2.8), we get

$$
\left\|u-v_{m}\right\|_{V}^{2}-\int_{\Gamma_{f}} S \gamma\left(\left|u_{\tau}\right|\right)\left(\left|u_{\tau}\right|-\left|v_{m \tau}\right|\right)^{2} \leq 2 a \int_{\Gamma_{f}} S|| v_{m \tau}|-| u_{\tau}||^{3} .
$$

Since the trace map is continuous from $V$ to $L^{3}\left(\Gamma_{f}\right)$ the last inequality becomes

$$
\left\|u-v_{m}\right\|_{V}^{2}-c_{u} \int_{\Gamma_{f}}\left(\left|u_{\tau}\right|-\left|v_{m \tau}\right|\right)^{2} \leq C\left\|u-v_{m}\right\|_{V}^{3},
$$

where $C$ is a generic constant. If $c_{u} \leq 0$, then we obtain $1 \leq C\left\|u-v_{m}\right\|_{V}$, a contradiction. If $c_{u}>0$, then from (3.11) and (3.16) we get $\frac{\lambda_{1}-c_{u}}{\lambda_{1}}\left\|u-v_{m}\right\|_{V}^{2} \leq C\left\|u-v_{m}\right\|_{V}^{3}$ which implies $\lambda_{1}-c_{u} \leq C \lambda_{1}\left\|\bar{u}-v_{m}\right\|_{V}$. Since $v_{m} \rightarrow u$ we obtain $\lambda_{1}-c_{u} \leq 0$, which contradicts (3.14).

4. The perturbed problem. Denote it by $\mathbb{R}_{+}^{3}=\left\{x \in \mathbb{R}^{3} ; x_{3}>0\right\}$ and $\mathbb{R}_{-}^{3}=$ $\left\{x \in \mathbb{R}^{3} ; x_{3}<0\right\}$.

Throughout the paper we will use $M$ as an arbitrary constant independent of any parameter. Also by $B_{1}^{2}(0)$ we denote the two-dimensional ball centered in 0 and with radius 1 .

Let $\Gamma_{f}^{0} \subset \Sigma_{0}$, with $\operatorname{dist}\left(\Gamma_{f}^{0}, \Sigma_{0}\right)>0$, be the unperturbed (or equivalent) fault and let $\epsilon>0$ be a small parameter. Let $T$ be a fixed open set compactly inclosed in the 2-dimensional unit square and consider the lattice $\frac{\epsilon}{2} \mathbf{Z}^{2}$ on the plane $\Pi$. Let

$$
Q_{i, 2}^{\epsilon}=\left\{x_{i}^{\epsilon}+\left(-\frac{\epsilon}{2}, \frac{\epsilon}{2}\right)^{2}\right\} \times\{0\}
$$

denote the periodic cell on $\Pi$, centered at $x=\left(x_{i}^{\epsilon}, 0\right)$, where $x_{i}^{\epsilon}=i \epsilon$ for $i \in \mathbb{Z}^{2}$.

The perturbed fault $\Gamma_{f}^{\epsilon} \subset \Sigma_{0}$ has $\epsilon$-periodically distributed holes, called (small-scale) barriers. More precisely in each $\epsilon$-square of the $\epsilon$-lattice on the fault plane $\Pi$, the friction contact is considered outside an open set $T_{\epsilon}$ (small-scale barrier) of size $r_{\epsilon}<\epsilon$ (see Figure 2 ) with $T_{\epsilon}=r_{\epsilon} T+\frac{k}{2} \epsilon, k \in \mathbf{Z}^{2}$. For the simplicity of the exposition we will assume that $T_{\epsilon}$ is a 2-dimensional ball, denoted by $B^{2}\left(x_{i}^{\epsilon}, r_{\epsilon}\right)$ centered in $x_{i}^{\epsilon}, i \in \mathbf{Z}^{2}$, and of radius $r_{\epsilon}$. 
We shall denote by $B_{\epsilon}$ the set of all the microscopic barriers and let $\Gamma_{f}^{\epsilon}:=\Gamma_{f}^{0} \backslash \bar{B}_{\epsilon}$ be the perturbed fault. As before we define $\Gamma_{t}^{\epsilon}:=\Sigma_{0} \backslash \Gamma_{f}^{\epsilon}$.

Now define the spaces

$$
X=\left\{u \in\left[H^{1}(\Omega)\right]^{3} / u=0 \text { on } \Gamma_{d}\right\}, \quad V=\left\{u \in X / u_{\tau}=0 \text { on } \Sigma \backslash \Gamma_{f}^{0}\right\}
$$

and let $W=V_{1}^{\perp}$ be the orthogonal complement of $V_{1}$ in $V$.

We define the perturbed problem

find $u^{\epsilon}: \Omega \rightarrow \mathbb{R}^{3}$, the solution of (2.15)-(2.17) with $\Gamma_{f}=\Gamma_{f}^{\epsilon}$ and $\Gamma_{t}=\Gamma_{t}^{\epsilon} . \quad\left(\left(\mathcal{P}_{\epsilon}\right)\right)$

We consider

$$
V_{\epsilon}:=\left\{v \in\left[H^{1}(\Omega)\right]^{3} / v=0 \quad \text { on } \quad \Gamma_{d}, \quad v_{\tau}=0 \quad \text { on } \Gamma_{t}^{\epsilon}\right\}
$$

to formulate $\left(\mathcal{P}_{\epsilon}\right)$ in terms of the minimum of energy, $\mathcal{W}_{\epsilon}: V_{\epsilon} \rightarrow \mathbb{R}$, i.e.

$$
u_{\epsilon} \in V_{\epsilon}, \quad \mathcal{W}_{\epsilon}\left(u_{\epsilon}\right) \leq \mathcal{W}_{\epsilon}(v), \quad \forall v \in V_{\epsilon} .
$$

We define the perturbed eigenvalue problem, associated to the above perturbed minimum problem, as

find $u^{\epsilon}: \Omega \rightarrow \mathbb{R}^{3}$, and $\lambda^{\epsilon}$, the solution of (3.7)- with $\Gamma_{f}=\Gamma_{f}^{\epsilon}$ and $\Gamma_{t}=\Gamma_{t}^{\epsilon}$,

which has the variational formulation

$$
u^{\epsilon} \in V_{\epsilon}, \quad\left\langle u^{\epsilon}, v\right\rangle_{V}=\lambda^{\epsilon} \int_{\Gamma_{f}^{0}} u_{\tau}^{\epsilon} \cdot v_{\tau}, \quad \forall v \in V_{\epsilon} .
$$

Let $L^{\epsilon}=\left\{f=\left(f_{1}, f_{2}, 0\right) / f_{1}, f_{2} \in L^{2}\left(\Sigma_{0}\right), f_{1}=f_{2}=0\right.$ on $\left.\Gamma_{t}^{\epsilon}\right\}$ and let the tangential trace on $\Gamma_{f}^{\epsilon}$ be defined as before. Thus if we consider

$$
V_{1}^{\epsilon}=\left\{v \in V_{\epsilon} ; v_{\tau}=0 \text { on } \Gamma_{f}^{\epsilon}\right\}
$$

we can see that

$$
V_{1}^{\epsilon}=V_{1}=\left\{v \in V ; \quad v_{\tau}=0 \quad \text { on } \Sigma_{0}\right\},
$$

and $V_{1}$ is a subspace of $V_{\epsilon}$. Let us define $W_{\epsilon} \doteq V_{1}^{\perp}$ to be the orthogonal complement of $V_{1}$ in $V_{\epsilon}$, and $P_{W_{\epsilon}}: V_{\epsilon} \rightarrow W_{\epsilon}$ to be the orthogonal projection onto $W_{\epsilon}$.

Then as is the proof of Theorem 3.2 we can write (4.4) as an eigenvalue problem for the operator $K^{\epsilon}: W_{\epsilon} \rightarrow W_{\epsilon}$, defined by

$$
\left\langle K^{\epsilon} u, v\right\rangle=\int_{\Gamma_{f}^{0}} u_{\tau} v_{\tau} .
$$

Thus the problem (4.4) will have an orthonormal sequence of eigenvectors $\left\{u_{n}^{\epsilon}\right\}_{n \geq 1}$ and a sequence of corresponding eigenvalues $\left\{\lambda_{n}^{\epsilon}\right\}_{n \geq 1}$, such that $0<\lambda_{1}^{\epsilon} \leq \lambda_{2}^{\epsilon} \leq \ldots$, $\lambda_{n}^{\epsilon} \longrightarrow+\infty$, and

$$
\frac{\left\|u_{1}^{\epsilon}\right\|_{V}^{2}}{\int_{\Gamma_{f}^{\epsilon}}\left|u_{1 \tau}^{\epsilon}\right|^{2} d x}=\lambda_{1}^{\epsilon}=\min _{v \in W_{\epsilon}} \frac{\|v\|_{V}^{2}}{\int_{\Gamma_{f}^{\epsilon}}\left|v_{\tau}\right|^{2} d x}
$$




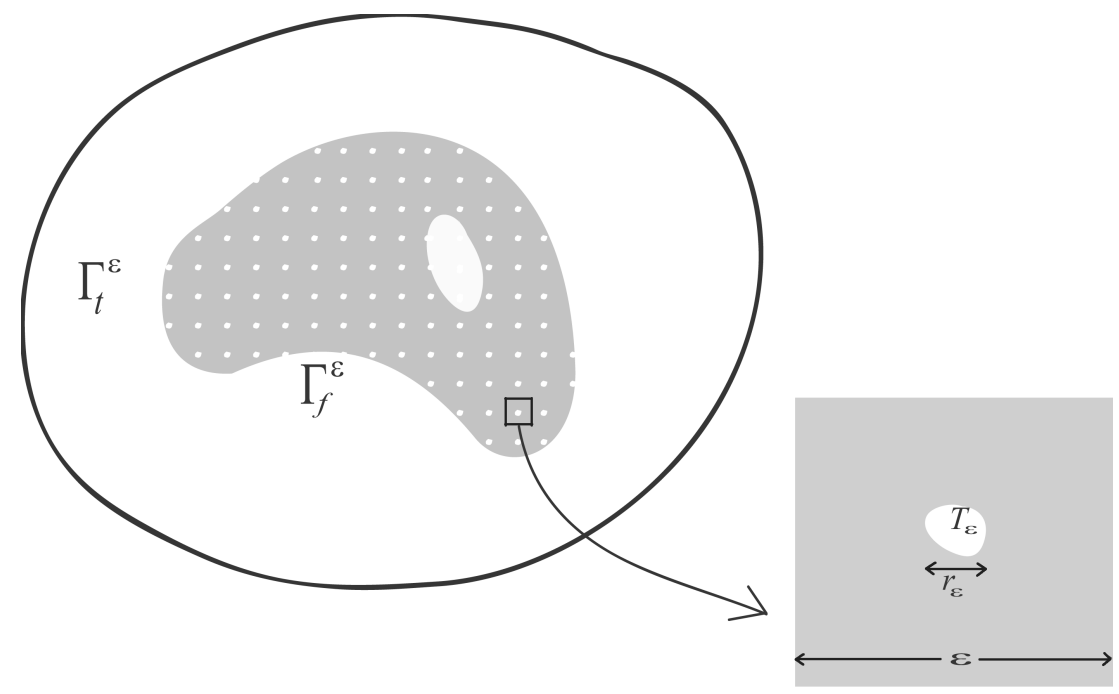

FIG. 2. The splitting of $\Sigma_{0}$ into $\Gamma_{f}^{\epsilon}$ (the friction surface) in grey and $\Gamma_{t}^{\epsilon}$ (the barrier surface) in white for the perturbed problem.

4.1. Asymptotic analysis of the problem $\left(\mathcal{P}_{\epsilon}\right)$. The main theorem concerning the homogenization of problem $\left(\mathcal{P}_{\epsilon}\right)$ is given next:

TheOrem 4.1. The sequence of functionals $\mathcal{W}_{\epsilon}: V_{\epsilon} \rightarrow \mathbb{R}$

$$
\mathcal{W}_{\epsilon}(v)=\frac{1}{2}\|v\|_{V}+\int_{\Gamma_{f}^{0}} S H\left(\left|v_{\tau}\right|\right)-f(v)
$$

$\Gamma$-converge with respect to the weak topology of $V$ to, $\overline{\mathcal{W}}: V \rightarrow \mathbb{R}$ with

$$
\overline{\mathcal{W}}(v)=\frac{1}{2}\|v\|_{V}^{2}+\int_{\Gamma_{f}^{0}} S H\left(\left|v_{\tau}\right|\right)-f(v)+\frac{1}{2} c \sum_{i, j=1}^{3} \int_{\Gamma_{f}^{0}} C_{i j} v_{i} v_{j},
$$

where for $k, l=\overline{1,3}$,

$$
C_{k l}= \begin{cases}0 & \text { if }(k-3)(l-3)=0, \\ \int_{\mathbb{R}_{+}^{3}} \mathcal{A} \epsilon\left(w^{k}\right) \epsilon\left(w^{l}\right) d x & \text { otherwise }\end{cases}
$$

$0<c=\lim _{\epsilon \rightarrow 0} \frac{r_{\epsilon}}{\epsilon^{2}}<\infty$ and $w^{k}$, for $k=\overline{1,2}$, is the solution of the following local problem:

$$
\begin{cases}\frac{\partial}{\partial y_{j}} \sigma_{i j}\left(w^{k}\right)=0 & \text { on } \mathbb{R}_{+}^{3} \text { for } i=1,2,3, \\ \sigma_{33}\left(w^{k}\right)=0, w_{\tau}^{k}=e^{k} & \text { on } B_{1}^{2}(0), \\ \sigma_{i 3}\left(w^{k}\right)=0 & \text { on } \mathbb{R}^{2} \backslash B_{1}^{2}(0), \\ w^{k}(y) \rightarrow 0 & \text { where } y_{3} \geq 0 \text { and }|y| \rightarrow \infty .\end{cases}
$$

Before beginning the proof we make the following useful remark. 
REMARK 4.2. The result above can be rewritten in the following way: $\Gamma-\lim _{\epsilon \rightarrow 0} \mathcal{W}_{\epsilon}=$ $\overline{\mathcal{W}}$, where $\overline{\mathcal{W}}: V \rightarrow \mathbb{R}$ is defined as

$$
\overline{\mathcal{W}}(v)=\frac{1}{2}\|v\|_{V}^{2}+\int_{\Gamma_{f}^{0}} S H\left(\left|v_{\tau}\right|\right)-f(v)+\frac{1}{2} c \int_{\Gamma_{f}^{0}} v_{\tau} C v_{\tau}
$$

with $c$ and the matrix $C$ defined as above.

Proof. We mention that the asymptotic analysis of this problem uses similar techniques as those developed in [2] and [3]. For the convenience of the reader we present the proof of our results, referring to the above-mentioned papers when needed. The next lemma can be easily adapted from [2] using Korn's inequality.

Lemma 4.3. Let $\left(u_{j}\right)$ be bounded in $V$ and let $N, k \in \mathbb{N}$. Let $\epsilon_{j}$ be a decreasing sequence of positive numbers converging to 0 and let

$$
\mathbb{Z}_{f, 1}^{j}=\left\{i \in \mathbb{Z}^{2} / B^{2}\left(x_{i}^{\epsilon_{j}}, r_{\epsilon_{j}}\right) \cap \Gamma_{f}^{0} \neq \emptyset\right\} \text { and } \mathbb{Z}_{f}^{j}=\left\{i \in \mathbb{Z}^{2} / Q_{i, 2}^{\epsilon_{j}} \subset \Gamma_{f}^{0}\right\} .
$$

Let $\left(\rho_{\epsilon_{j}}\right)$ be a sequence of positive numbers, such that $N \rho_{\epsilon_{j}}<\frac{1}{2} \epsilon_{j}$. For all $i \in \mathbb{Z}_{f}^{j}$ there exists $k_{i} \in\{0, \ldots, k-1\}$ such that, having set

$$
C_{i}^{j}=\left\{x \in \mathbb{R}_{+}^{3} / 2^{-k_{i}-1} N \rho_{\epsilon_{j}}<\left|x-\left(x_{i}^{\epsilon}, 0\right)\right|<2^{-k_{i}} N \rho_{\epsilon_{j}}\right\},
$$

$u_{j}^{i}=\frac{1}{\left|C_{i}^{j}\right|} \int_{C_{i}^{j}} u_{j} d x$ (the mean value of $u_{j}$ on $C_{i}^{j}$ ) and $\rho_{i}^{j}=\frac{3}{4} 2^{-k_{i}} N \rho_{\epsilon_{j}}$ (the middle radius of $\left.C_{i}^{j}\right)$, there exists a sequence $\left(w_{j}\right)$ such that

$$
w_{j}=u_{j} \text { on } \Omega \backslash \bigcup_{i \in \mathbb{Z}_{f}^{j}} C_{i}^{j}
$$

$w_{j}(x)=u_{j}^{i}$ if $\left|x-\left(x_{i}^{\epsilon}, 0\right)\right|=\rho_{j}^{i}$ and $x_{3}>0$, for $i \in \mathbb{Z}_{f}^{j}$, and

$$
\sum_{i \in \mathbb{Z}_{f}^{j}} \int_{C_{i}^{j}}\left(\mathcal{A} \epsilon\left(w_{j}\right) \epsilon\left(w_{j}\right)+\mathcal{A} \epsilon\left(u_{j}\right) \epsilon\left(u_{j}\right) d x\right) \leq \frac{M}{k},
$$

where $M$ is independent of $j$.

Moreover, if $\rho_{\epsilon_{j}}^{3}=o\left(\epsilon_{j}^{2}\right)$, and the sequence $\left(\left|D u_{j}\right|^{2}\right)$ is equi-integrable in $\Omega$, then we can choose $k_{i}=0$ for all $i \in \mathbb{Z}_{f}^{j}$ and

$$
\lim _{j \rightarrow+\infty} \sum_{i \in \mathbb{Z}_{f}^{j}} \int_{C_{i}^{j}}\left(\mathcal{A} \epsilon\left(w_{j}\right) \epsilon\left(w_{j}\right)+\mathcal{A} \epsilon\left(u_{j}\right) \epsilon\left(u_{j}\right)\right)=0 .
$$

As in 2] we can make the following remark.

REMARK 4.4. If $u_{j} \rightarrow u$ strongly in $L^{2}(\Omega)$ and $\sup \mathcal{W}_{\epsilon_{j}}\left(u_{j}\right)<+\infty$, then $u_{j} \rightarrow u$ weakly in $V$. Moreover if $\left(w_{j}\right)$ is defined as in the above lemma, then $w_{j} \rightarrow u$ strongly in $L^{2}(\Omega)$ and since $\left(w_{j}\right)$ is bounded in $V$, we also get that $\left(w_{j}\right)$ converges weakly to $u$ in $V$. If $\left(\left|D u_{j}\right|^{2}\right)$ is equi-integrable, then $\left(\left|D w_{j}\right|^{2}\right)$ is also equi-integrable.

For any $R \in \mathbb{R}_{+}$we will denote by $B_{R}(x) \subset \mathbb{R}^{3}$ the ball centered in $x \in \mathbb{R}^{3}$, and radius $R$, and $B_{R}^{+}(x)=B_{R}(x) \cap \mathbb{R}_{+}^{3}$. 
Let

$$
\begin{aligned}
\phi_{N}(z)=\inf \left\{\int_{B_{N}^{+}(0)} \mathcal{A} \epsilon(v) \epsilon(v) /\right. & v \in H^{1}\left(B_{N}^{+}(0), \mathbb{R}^{3}\right), \\
& \left.v_{\tau}=0 \text { on } B_{1}^{2}(0), v=z \text { on } \partial B_{N}^{+}(0) \backslash \Sigma_{0}\right\} .
\end{aligned}
$$

We will now make another useful remark:

REMARK 4.5. If $f$ is a convex function and $0 \leq f(A) \leq M\left(1+|A|^{2}\right)$, then $f$ is locally Lipschitz, i.e.

$$
|f(A)-f(B)| \leq M(1+|A|+|B|)|A-B|, \quad \text { for all } A, B \in \mathcal{M}^{3 \times 3} .
$$

In addition if $f$ is also homogeneous of order 2 , then

$$
|f(A)-f(B)| \leq M(|A|+|B|)|A-B| \quad \text { for all } A, B \in \mathcal{M}^{3 \times 3} .
$$

Proof. Indeed from (4.7) if we consider a sequence $\epsilon_{j}$, such that $\epsilon_{j} \rightarrow 0$, we have

$$
|f(A)-f(B)|=\epsilon_{j}^{2}\left|f\left(\frac{1}{\epsilon_{j}} A\right)-f\left(\frac{1}{\epsilon_{j}} B\right)\right| \leq \epsilon_{j}^{2} M\left(1+\frac{1}{\epsilon_{j}}|A|+\frac{1}{\epsilon_{j}}|B|\right) \frac{1}{\epsilon_{j}}|A-B| .
$$

Thus

$$
|f(A)-f(B)| \leq M \epsilon_{j}|A-B|+M(|A|+|B|)|A-B|
$$

for all $A, B \in \mathcal{M}^{3 \times 3}$ and for any $j \in \mathbb{N}$. Then passing to the limit where $j \rightarrow \infty$, we have (4.8).

Now we have

Lemma 4.6. For all $N \in \mathbb{N}$ with $N>2, \phi_{N}$ defined above verifies

$$
\left|\phi_{N}(z)-\phi_{N}(w)\right| \leq M|w-z|(|z|+|w|) \quad \text { for all } z, w \in \mathbb{R}^{3} .
$$

Proof. Fix $1>\nu>0$. Using the definition of $\phi_{N}(z)$ we find $\bar{w} \in H^{1}\left(B_{N}^{+}(0) ; \mathbb{R}^{3}\right)$, with $\bar{w}=0$ on $\partial B_{N}^{+}(0) \backslash \Sigma_{0}$ and $\bar{w}_{\tau}=-z_{\tau}$ on $B_{1}^{2}(0)$ such that

$$
\int_{B_{N}^{+}(0)} \mathcal{A} \epsilon(\bar{w}) \epsilon(\bar{w}) \leq \phi_{N}(z)+\nu
$$

Let $\varphi \in C_{0}^{\infty}\left(B_{2}(0)\right)$ be a cutoff function such that $\phi=1$ on $B_{1}(0)$ and $|D \varphi| \leq M$. For $w \in \mathbb{R}^{3}$ define $\psi=\bar{w}+(1-\varphi)(w-z)$ on $B_{N}^{+}(0)$. Then we can see that

$$
\begin{cases}\psi_{\tau}=-z_{\tau} & \text { on } B_{1}^{2}(0) \\ \psi=w-z & \text { on } \partial B_{N}^{+}(0) \backslash \Sigma_{0}\end{cases}
$$

so $\psi$ is a test function for $\phi_{N}(w)$. Thus using (2.3), (4.8) and (4.6) we have

$$
\begin{aligned}
\phi_{N}(w)-\phi_{N}(z) & \leq \int_{B_{N}^{+}(0)} \mathcal{A} \epsilon(\psi) \epsilon(\psi) d x-\int_{B_{N}^{+}(0)} \mathcal{A} \epsilon(\bar{w}) \epsilon(\bar{w})+\nu \\
& \leq M \int_{B_{N}^{+}(0)}(|\epsilon(\psi)|+|\epsilon(\bar{w})|) \cdot(|\epsilon(\psi)-\epsilon(\bar{w})|) d x+\nu
\end{aligned}
$$


Next from the definition of the test function $\psi$ in (4.11) we obtain

$$
\begin{aligned}
\phi_{N}(w)-\phi_{N}(z) & \leq M \int_{B_{N}^{+}(0)}(2|\epsilon(\bar{w})|+|w-z||D \varphi|)|w-z||D \varphi|+\nu \\
& \leq M|w-z|\left(\int_{B_{N}^{+}(0)}|\epsilon(\bar{w})|^{2} d x\right)^{1 / 2} \cdot\left(\int_{B_{N}^{+}(0)}|D \varphi|^{2} d x\right)^{1 / 2} \\
& +M|w-z|^{2} \int_{B_{N}^{+}(0)}|D \varphi|^{2} d x+\nu .
\end{aligned}
$$

Since $N>2$ we have that $\int_{B_{N}^{+}(0)}|D \varphi| d x$ and $\int_{B_{N}^{+}(0)}|D \varphi|^{2} d x$ are constants independent of $N$, and by condition (2.3) and the definition of $\phi_{N}$ we get

$$
M \int_{B_{N}^{+}(0)}|\epsilon(\bar{w})|^{2} d x \leq \int_{B_{N}^{+}(0)} \mathcal{A} \epsilon(\bar{w}) \epsilon(\bar{w}) \leq \phi_{N}(z)+\nu
$$

and from Korn's inequality and monotonicity of $\phi_{N}$

$$
\begin{gathered}
\phi_{N}(z) \leq \phi_{2}(z) \leq M|z|^{2} \inf \left\{\int_{B_{2}^{+}(0)}|D v|^{2} d x v \in H^{1}\left(B_{2}^{+}(0) ; \mathbb{R}^{3}\right),\right. \\
\left.v_{\tau}=0 \quad \text { on } B_{1}^{2}(0), \quad v=\frac{z}{|z|} \text { on } \partial B_{2}^{+}(0) \backslash \Sigma_{0}\right\} .
\end{gathered}
$$

But

$$
\left\{v / v \in H^{1}\left(B_{2}^{+}(0) ; \mathbb{R}^{3}\right), v_{\tau}=0 \text { on } B_{1}^{2}(0), v=\frac{z}{|z|} \text { on } \partial B_{2}^{+}(0) \backslash \Sigma_{0}\right\}
$$

contains

$$
\left\{v / v \in H^{1}\left(B_{2}(0) \backslash C_{1,2} ; \mathbb{R}^{3}\right), v=\frac{z}{|z|} \text { on } \partial B_{2}^{+}(0) \backslash \Sigma_{0}, v=0 \text { on } B_{2}(0) \cap \mathbb{R}_{-}^{3}\right\}
$$

as a subset, where

$$
C_{1,2}=\left\{\left(x^{\prime}, 0\right) \in \mathbb{R}^{3}: 1 \leq\left|x^{\prime}\right|<2\right\} .
$$

From (4.14) and the above inclusion we obtain

$$
\begin{gathered}
\phi_{N}(z) \leq \phi_{2}(z) \leq M|z|^{2} \inf \left\{\int_{B_{2}^{+}(0)}|D v|^{2} d x / v \in H^{1}\left(B_{2}(0) \backslash C_{1,2}, \mathbb{R}^{3}\right),\right. \\
\left.v=\frac{z}{|z|} \text { on } \partial B_{2}^{+}(0) \backslash \Sigma_{0}, v=0 \text { on } B_{2}(0) \cap \mathbb{R}_{-}^{3}\right\} .
\end{gathered}
$$

Now following the ideas in 2] (see (4.9)) we obtain

$$
\phi_{N}(z) \leq M \frac{|z|^{2}}{2} \operatorname{Cap}\left(B_{1}^{2}(0)\right)
$$

where $\operatorname{Cap}\left(B_{1}^{2}(0)\right)$ is the usual capacity, i.e.

$$
\operatorname{Cap}\left(B_{1}^{2}(0)\right)=\inf \left\{\int_{\mathbb{R}^{3}}|D \phi|^{2} d x, \phi \in H^{1}\left(\mathbb{R}^{3}\right), \phi=1 \text { on } B_{1}^{2}(0)\right\} .
$$

By (4.13) we get

$$
\int_{B_{N}^{+}(0)}|\epsilon(\bar{w})|^{2} d x \leq M\left(\frac{|z|^{2}}{2}+\nu\right) .
$$


Now, from the results obtained so far and from (4.15) and (4.12), we have

$$
\begin{gathered}
\phi_{N}(w)-\phi_{N}(z) \leq M|w-z|\left(\frac{|z|^{2}}{2}+\nu\right)^{1 / 2}+M|w-z|^{2}+\nu \\
\leq M|w-z|((\nu+1)|z|+|w|+\sqrt{\nu})+\nu
\end{gathered}
$$

Now by the arbitrariness of $\nu$ we get that

$$
\phi_{N}(w)-\phi_{N}(z) \leq M|w-z|(|z|+|w|) .
$$

Lemma 4.7. $\phi_{N} \rightarrow \phi$ uniformly, where $\phi(z)=\sum_{k, l=1}^{3} C_{k l} z_{k} z_{l}$, and $C_{k l}$ is given by (4.16) and the local problem $(L P)$.

Proof. From Ascoli-Arzela's Theorem we have that $\phi_{N} \rightarrow \phi$ uniformly on compact sets of $\mathbb{R}^{3}$.

For any $N \in \mathbb{N}$ the problem (4.9) has a unique solution $\tilde{w}+z$ for fixed $z \in \mathbb{R}^{3}$. The Euler-Lagrange equation for $\tilde{w}$ is

$$
\begin{cases}\sigma(\tilde{w}=\mathcal{A} \epsilon(\tilde{w}),-\operatorname{div} \sigma(\tilde{w})=0 & \text { on } B_{N}^{+}(0), \\ \sigma_{33}(\tilde{w})=0, \tilde{w}_{\tau}=-z_{\tau} & \text { on } B_{1}^{2}(0), \\ \sigma_{i j}(\tilde{w}) n_{j}=0 & \text { on } C_{1, N}, \\ \tilde{w}=0 & \text { on } \partial B_{N}^{+}(0) \backslash \Sigma_{0} .\end{cases}
$$

So $\phi_{N}=\int_{B_{N}^{+}(0)} \mathcal{A} \epsilon(\tilde{w}) \epsilon(\tilde{w}) d x=\sum_{k, l=1}^{3} C_{k l}^{N} z_{k} z_{l}$, where

$$
C_{k l}^{N}=\left\{\begin{array}{ll}
0 & (k-3)(l-3)=0, \\
\int_{B_{N}^{+}(0)} \mathcal{A} \epsilon\left(w_{N}^{k}\right) \epsilon\left(w_{N}^{l}\right) & \text { otherwise, }
\end{array} \quad \text { for } k, l=\overline{1,3},\right.
$$

where $w_{N}^{k}$ for $k=\overline{1,2}$ is the solution of the local problem

$$
\begin{cases}\frac{\partial}{\partial y_{j}} \sigma_{i j}\left(w_{N}^{k}\right)=0 & \text { on } B_{N}^{+}(0), \quad \text { for } i=\overline{1,3}, \\ \sigma_{33}\left(w_{N}^{k}\right)=0, w_{N \tau}^{k}=e^{k} & \text { on } B_{1}^{2}(0), \\ \sigma_{i 3}\left(w_{N}^{k}\right)=0 & \text { on } C_{1, N}, \\ w_{N}^{k}=0 & \text { on } \partial B_{N}^{+}(0) \backslash \Sigma,\end{cases}
$$

where $\left\{e^{k}\right\}_{k=1,3}$ is the canonical base of $\mathbb{R}^{3}$. Therefore $\phi_{N} \rightarrow \phi$ uniformly on compacts subsets of $\mathbb{R}^{3}$ where

$$
\begin{aligned}
& \phi(z)=\sum_{k, l=1}^{3} C_{k l} z_{k} z_{l} \text { and } \\
& C_{k l}=\left\{\begin{array}{ll}
0 & (k-3)(l-3)=0, \\
\int_{\mathbb{R}_{+}^{3}}^{\mathcal{A}} \mathcal{A}\left(w^{k}\right) \epsilon\left(w^{l}\right) d x & \text { otherwise, }
\end{array} \text { for } k, l=\overline{1,3},\right.
\end{aligned}
$$


and $w^{k}$ for $k=\overline{1,2}$ is the solution of the local problem

$$
(L P) \begin{cases}\frac{\partial}{\partial y_{j}} \sigma_{i j}\left(w^{k}\right)=0 & \text { on } \mathbb{R}_{+}^{3} \text { for } i=\overline{1,3}, \\ \sigma_{33}\left(w^{k}\right)=0, w_{\tau}^{k}=e^{k} & \text { on } B_{1}^{2}(0), \\ \sigma_{i 3}\left(w^{k}\right)=0 & \text { on } \mathbb{R}^{2}-B_{1}^{2}(0), \\ w^{k}(y) \rightarrow 0 & \text { when }|y| \rightarrow \infty .\end{cases}
$$

REMARK 4.8. From Lemma 4.7 we can see that

$$
\phi_{N}(z)=\phi_{N}\left(z_{\tau}\right) \text { and } \phi(z)=\phi\left(z_{\tau}\right) .
$$

Now using Remark 4.8, by similar techniques as in [2, Prop. 4.4] we have

Lemma 4.9. Let $u_{j} \rightarrow u$ weakly in $V$ and bounded in $L^{\infty}\left(\Omega ; \mathbb{R}^{3}\right)$. Consider $\psi_{j}$ to be defined as

$$
\psi_{j}=\sum_{i \in \mathbb{Z}_{f}^{j}} \phi_{N}\left(u_{j \tau}^{i}\right) \chi_{Q_{i, 2}^{\epsilon_{j}}}
$$

where

$$
Q_{i, 2}^{\epsilon_{j}}=\left(x_{i}^{\epsilon_{j}}, 0\right)+\left(-\frac{\epsilon_{j}}{2}, \frac{\epsilon_{j}}{2}\right)^{2},
$$

and $u_{j}^{i}$ and $\mathbb{Z}_{f}^{j}$ are defined in Lemma 4.3 .

Then we have

$$
\lim _{j \rightarrow \infty} \int_{\Gamma_{f}^{0}}\left|\psi_{j}-\phi_{N}\left(u_{\tau}\right)\right| d s=0 .
$$

Proof. First we will show that

$$
\left|\Gamma_{f}^{0} \backslash \bigcup_{i \in \mathbb{Z}_{f}^{j}} Q_{i, 2}^{\epsilon_{j}}\right| \stackrel{j \rightarrow \infty}{\longrightarrow} 0 .
$$

Indeed, let

$$
w_{j}^{\prime \prime}=\bigcup_{\substack{i \in \mathbb{Z}^{2} \backslash \mathbb{Z}_{f}^{j} \\ \epsilon_{j}}} Q_{i, 2}^{\epsilon_{j}} .
$$

Then it can easily be seen that

$$
\left(\Gamma_{f}^{0} \backslash \bigcup_{i \in \mathbb{Z}_{f}^{j}} Q_{i, 2}^{\epsilon_{j}}\right) \subset w_{j}^{\prime \prime}
$$

and therefore we obtain, when $j \rightarrow \infty$, that

$$
\lim _{j \rightarrow \infty}\left|\Gamma_{f}^{0} \backslash \bigcup_{i \in \mathbb{Z}_{f}^{j}} Q_{i, 2}^{\epsilon_{j}}\right| \leq \lim _{j \rightarrow \infty} \mathcal{H}^{2}\left(w_{j}^{\prime \prime}\right) \leq \mathcal{H}^{2}\left(\partial \Gamma_{f}^{0}\right)=0 .
$$


Using Remark 4.8 we get

$$
\begin{aligned}
& \lim _{j \rightarrow \infty} \int_{\Gamma_{f}^{0}}\left|\psi_{j}-\phi_{N}\left(u_{\tau}\right)\right| d s=\lim _{j \rightarrow \infty} \int_{\Gamma_{f}^{0}}\left|\sum_{i \in \mathbb{Z}_{f}^{j}} \phi_{N}\left(u_{j}^{i}\right) \chi_{Q_{i, 2}^{\epsilon_{j}}}-\phi_{N}(u)\right| \\
& \leq \lim _{j \rightarrow \infty}\left(\sum_{i \in \mathbb{Z}_{f}^{j}} \int_{Q_{i, 2}^{\epsilon_{j}}}\left|\phi_{N}\left(u_{j}^{i}\right)-\phi_{N}(u)\right| d s\right)+\lim _{j \rightarrow \infty} \int_{\Gamma_{f}^{0} \backslash \cup_{i \in \mathbb{Z}_{f}^{j}} Q_{i, 2}^{\epsilon_{j}}}\left|\phi_{N}(u)\right| .
\end{aligned}
$$

Now from Lemma 4.6 and (4.17), the uniform boundedness of $\phi_{N}$, Lemma 4.6 and the boundedness of $\left(u_{j}\right)_{j}$ in $L^{\infty}\left(\Omega, \mathbb{R}^{3}\right)$, we get

$$
\lim _{j \rightarrow \infty} \int_{\Gamma_{f}^{0}}\left|\psi_{j}-\phi_{N}\left(u_{\tau}\right)\right| d s \leq M \lim _{j \rightarrow \infty} \sum_{i \in \mathbb{Z}_{f}^{j}} \int_{Q_{i, 2}^{\epsilon_{j}}}\left|u_{j}^{i}-u\right| d s .
$$

By similar arguments as in [2, Prop. 4.4], we can prove that

$$
\lim _{j \rightarrow \infty} \sum_{i \in \mathbb{Z}_{f}^{j}} \int_{Q_{i, 2}^{\epsilon_{j}}}\left|u_{j}^{i}-u\right| d s=0,
$$

and this proves the statement of the lemma.

Using Remark 4.8, Lemma 4.7, Lemma 4.9, by similar arguments as in [2, Sec. 5], we obtain the liminf inequality

Lemma 4.10. Consider $\rho_{\epsilon_{j}} \doteq r_{\epsilon_{j}}$ defined as in Lemma 4.3 such that

$$
0<\lim _{j \rightarrow \infty} \frac{r_{\epsilon_{j}}}{\epsilon_{j}^{2}}=c<+\infty
$$

Let $k, N \in M$ fixed, and $N>2^{k}$. Then for any sequence $\left(u_{j}\right)_{j}$, such that $u_{j} \in V_{\epsilon_{j}}$ and $u_{j} \rightarrow u$ weakly in $V$, we have

$$
\liminf _{j \rightarrow \infty}\left\|u_{j}\right\|_{V}^{2} \geq\|u\|_{V}^{2}+c \sum_{k, l=1}^{3} \int_{\Gamma_{f}^{0}} C_{k l} u_{k} u_{l} d s,
$$

where the matrix $\left(C_{k l}\right)_{k, l=\overline{1,3}}$ is defined in Theorem 4.1

Proof. Remark 4.8 is very important. Because of the property mentioned in Remark 4.8. we can follow the proof in [2, Sec. 5], although in our case the space $V_{\epsilon}$ is not the same as in 2] and therefore the functions $\phi_{N}$ and $\phi$ respectively are not the same as in [2].

Let $u \in V$ and consider $\left\{u_{j}\right\}_{j}$ such that $u_{j} \in V_{\epsilon_{j}}$ and $u_{j} \rightarrow u$. Let $w_{j}$ and $\rho_{j}^{i}$ be as in Lemma 4.3 and

$$
E_{j}=\bigcup_{i \in \mathbb{Z}_{f}^{j}} B_{i}^{j} \text { with } B_{i}^{j}=B_{\rho_{j}^{i}}\left(x_{i}^{\epsilon_{j}}, 0\right) \cap\left\{x \in \mathbb{R}^{3} \mid x_{3}>0\right\} \text { for all } i \in \mathbb{Z}_{f}^{j} .
$$

We have

$$
\liminf _{j \rightarrow \infty}\left\|u_{j}\right\|_{V}^{2} \geq \liminf _{j \rightarrow \infty} \int_{\Omega \backslash E_{j}} \mathcal{A} \epsilon\left(u_{j}\right) \epsilon\left(u_{j}\right) d x+\liminf _{j \rightarrow \infty} \int_{E_{j}} \mathcal{A} \epsilon\left(u_{j}\right) \epsilon\left(u_{j}\right) d x .
$$


Next we can see that

$$
\frac{M}{k}+\liminf _{j \rightarrow \infty} \int_{\Omega \backslash E_{j}} \mathcal{A} \epsilon\left(u_{j}\right) \epsilon\left(u_{j}\right) d x \geq\|u\|_{V}^{2} .
$$

The proof of (4.20) is identical to the proof in [2, Prop. 5.1] and therefore we will not present it here.

Now similarly as in [2] let us define, for fixed $j \in \mathbb{N}$ and $i \in \mathbb{Z}_{f}^{j}$,

$$
\varphi(x)= \begin{cases}w_{j}\left(\left(x_{i}^{\epsilon_{j}}, 0\right)+r_{\epsilon_{j}} x\right) & \text { if } x \in B_{\frac{3}{4} 2^{-k_{i} N}}^{+}(0), \\ u_{j}^{i} & \text { if } x \in B_{N}^{+}(0) \backslash B_{\frac{3}{4} 2^{-k_{i} N}}^{+}(0),\end{cases}
$$

where $k_{i} \in \overline{1, k-1}$ and $u_{j}^{i}$ are as in Lemma 4.3 .

As in 2] we have, by Lemma 4.3 .

$$
\frac{M}{k}+\liminf _{j \rightarrow \infty} \int_{E_{j}} \mathcal{A} \epsilon\left(u_{j}\right) \epsilon\left(u_{j}\right) d x \geq \liminf _{j \rightarrow \infty} \int_{E_{j}} \mathcal{A} \epsilon\left(w_{j}\right) \epsilon\left(w_{j}\right) d x
$$

and using $\varphi$, defined in (4.21), as a test function in the definition of $\phi_{N}$ (see [2,5.31]) we get

$$
\liminf _{j \rightarrow \infty} \int_{E_{j}} \mathcal{A} \epsilon\left(w_{j}\right) \epsilon\left(w_{j}\right) d x \geq \liminf _{j \rightarrow \infty} \frac{r_{\epsilon_{j}}}{\epsilon_{j}^{2}} \sum_{i \in \mathbb{Z}_{f}^{j}} \epsilon_{j}^{2} \phi_{N}\left(u_{j}^{i}\right) .
$$

By Remark 4.8 and the hypothesis (4.18) we obtain

$$
\liminf _{j \rightarrow \infty} \int_{E_{j}} \mathcal{A} \epsilon\left(w_{j}\right) \epsilon\left(w_{j}\right) d x \geq c \cdot \liminf _{j \rightarrow \infty} \sum_{i \in \mathbb{Z}_{f}^{j}} \epsilon_{j}^{2} \phi_{N}\left(u_{j \tau}^{i}\right) .
$$

Note now that for any $j \in \mathbf{N}$ we have

$$
\sum_{i \in \mathbb{Z}_{f}^{j}} \epsilon_{j}^{2} \phi_{N}\left(u_{j \tau}^{i}\right)=\int_{\Gamma_{f}^{0}} \psi_{j} d s
$$

where $\psi_{j}$ has been defined in Lemma 4.9. Combining (4.22), (4.23), (4.24) we obtain

$$
\frac{M}{k}+\liminf _{j \rightarrow \infty} \int_{E_{j}} \mathcal{A} \epsilon\left(u_{j}\right) \epsilon\left(u_{j}\right) d x \geq c \liminf _{j \rightarrow \infty} \int_{\Gamma_{f}^{0}} \psi_{j} d s
$$

and from (4.20) and (4.25) we obtain

$$
\frac{M}{k}+\liminf _{j \rightarrow \infty} \int_{\Omega} \mathcal{A} \epsilon\left(u_{j}\right) \epsilon\left(u_{j}\right) d x \geq\|u\|_{V}^{2}+c \liminf _{j \rightarrow \infty} \int_{\Gamma_{f}^{0}} \psi_{j} d s .
$$

From Lemma 4.7, Lemma 4.9 (4.26) and the arbitrariness of $k \in \mathbb{N}$, using Lemma 3.5 from [7] as in [2, Prop. 5.2], we can "remove" the $L^{\infty}\left(\Omega, \mathbb{R}^{3}\right)$ boundedness hypothesis from Lemma 4.9, and obtain the liminf inequality in a similar manner.

Next, we will prove the limsup inequality.

LEMma 4.11. Let $r_{\epsilon_{j}}$ be such that

$$
0<\lim _{j \rightarrow \infty} \frac{r_{\epsilon_{j}}}{\epsilon_{j}^{2}}=c<+\infty .
$$


Then for all $u \in V$ and for all $\delta>0$ there exists a sequence $u_{j} \in V_{\epsilon_{j}}$ converging to $u$, in the weak topology of $V$, such that

$$
\limsup _{j \rightarrow \infty}\left\|u_{j}\right\|_{V}^{2}-\delta \leq\|u\|_{V}^{2}+c \int_{\Gamma_{f}^{0}} \sum_{k, l=1}^{3} C_{k l} u_{k} u_{l} d s,
$$

where $\left(C_{k l}\right)_{k, l=\overline{1,3}}$ has been defined in Lemma 4.7

Proof. Without loss of generality we will assume $\delta$ small enough. Again Remark 4.8 allows us to follow the same arguments as in [2, Sec. 6].

Indeed, suppose first that $u \in L^{\infty}\left(\Omega, \mathbb{R}^{3}\right)$. Recall that $B_{N \rho_{\epsilon_{j}}} \equiv B_{N \rho_{\epsilon_{j}}}\left(x_{i}^{\epsilon_{j}}, 0\right)$ and $B_{N \rho_{\epsilon_{j}}}^{+}=B_{N \rho_{\epsilon_{j}}} \cap\left\{x_{3}>0\right\}$. From Lemma 4.3 for $u_{j} \equiv u$ and $\rho_{\epsilon_{j}}=\frac{4}{3} r_{\epsilon_{j}}$ and from the equi-integrability condition we obtain a sequence $\left(w_{j}\right)_{j}$ such that

$$
w_{j}=u_{j}^{i}=\frac{1}{\left|C_{i}^{j}\right|} \int_{C_{i}^{j}} u \text { on } \partial B_{N r_{\epsilon_{j}}}^{+} \backslash \Sigma_{0},
$$

whereby $|A|$ we denote the usual superficial measure supported by $A$. Define

$$
v_{j}=w_{j} \text { on } \Omega \backslash \bigcup_{i \in \mathbb{Z}_{f, 1}^{j}} B_{N r_{\epsilon_{j}}}^{+} .
$$

Then because $\left|\bigcup_{i \in \mathbb{Z}_{f, 1}^{j}} B_{N r_{\epsilon_{j}}}^{+}\right| \sim \frac{r_{\epsilon_{j}}^{3}}{\epsilon_{j}^{2}}$ and $w_{j} \rightarrow u$ weakly in $V$, we obtain that $v_{j} \rightarrow u$ weakly in $V$. We will define $v_{j}$ on $\bigcup_{i} B_{N r_{\epsilon_{j}}}^{+}$below.

Next, using similar arguments as in [2, Sec. 6] we get

$$
\limsup _{j \rightarrow \infty}\left\|v_{j}\right\|_{V}^{2} \leq\|u\|_{V}^{2}+\limsup _{j \rightarrow \infty} \int_{\bigcup_{i \in \mathbb{Z}_{f, 1}^{j}} B_{N r_{\epsilon_{j}}}} \mathcal{A} \epsilon\left(v_{j}\right) \epsilon\left(v_{j}\right) d x .
$$

From Lemma 4.7, we have that for any $\delta>0$, there is an $N_{0} \in \mathbb{N}$ such that

$$
\phi(z)-\frac{\delta}{2} \leq \phi_{N}(z) \leq \phi(z)+\frac{\delta}{2} \text { for any } z \text { with }|z| \leq m,
$$

and for any $N \geq N_{0}$, where $m=\|u\|_{L^{\infty}\left(\Omega, \mathbb{R}^{3}\right)}$.

By the definition of $\phi_{N}$ there is $w_{j}^{i} \in H^{1}\left(B_{N}^{+}(0) ; \mathbb{R}^{3}\right), w_{j \tau}^{i}=0$ on $B_{1}^{2}(0)$ and $w_{j}^{i}=u_{j}^{i}$ on $\partial B_{N}^{+}(0) \backslash \Sigma_{0}$, such that

$$
\int_{B_{N}^{+}(0)} \mathcal{A} \epsilon\left(w_{j}^{i}\right) \epsilon\left(w_{j}^{i}\right) d x \leq \phi_{N}\left(u_{j}^{i}\right)+\frac{\delta}{2} \leq \phi\left(u_{j}^{i}\right)+\delta=\phi\left(u_{j \tau}^{i}\right)+\delta,
$$

where we used Remark 4.8 for the last equality above. Next, similar to [2, Sec. 6], we define $v_{j}$ on $\bigcup_{i \in \mathbb{Z}_{f, 1}^{j}} B_{N \rho_{\epsilon_{j}}}$ to be

$$
v_{j}=w_{j}^{i}\left(\frac{x-\left(x_{i}^{\epsilon_{i}}, 0\right)}{r_{\epsilon_{j}}}\right) \text { on } B_{N r_{\epsilon_{j}}} \text { for } i \in \mathbb{Z}_{f}^{j}
$$

and

$$
v_{j}=h \cdot\left(\frac{x-\left(x_{i}^{\epsilon_{i}}, 0\right)}{r_{\epsilon_{j}}}\right) w_{j}(x) \text { on } B_{N r_{\epsilon_{j}}}^{+} \text {for } i \in \mathbb{Z}_{f, 1}^{j} \backslash \mathbb{Z}_{f}^{j},
$$

where $0 \leq h \leq 1$ is the same scalar function used in [2], i.e. $h=1$ on $\partial B_{N}^{+}(0) \backslash \Sigma_{0}$ and $h=0$ on $B_{1}^{2}(0)$. 
From (4.27), (4.31) and (4.32) we can see that $v_{j} \in V_{\epsilon_{j}}$ and from (4.30) we have that

$$
\int_{B_{N r_{\epsilon_{j}}}^{+}} \mathcal{A} \epsilon\left(v_{j}\right) \epsilon\left(v_{j}\right) d x=r_{\epsilon_{j}} \int_{B_{N}^{+}(0)} \mathcal{A} \epsilon\left(w_{j}^{i}\right) \epsilon\left(w_{j}^{i}\right) d x \leq \frac{r_{\epsilon_{j}}}{\epsilon_{j}^{2}}\left(\epsilon_{j}^{2} \phi\left(u_{j \tau}^{i}\right)+\epsilon_{j}^{2} \delta\right)
$$

for any $i \in \mathbb{Z}_{f}^{j}$.

Obviously we have

$$
\begin{aligned}
\limsup _{j \rightarrow \infty} \sum_{i \in \mathbb{Z}_{f, 1}^{j}} \int_{B_{N r_{\epsilon_{j}}}^{+}} \mathcal{A} \epsilon\left(v_{j}\right) \epsilon\left(v_{j}\right) d x & \leq \limsup _{j \rightarrow \infty} \sum_{i \in \mathbb{Z}_{f, 1}^{j} \backslash \mathbb{Z}_{f}^{j}} \int_{B_{N r_{\epsilon_{j}}}^{+}} \mathcal{A} \epsilon\left(v_{j}\right) \epsilon\left(v_{j}\right) d x \\
& +\limsup _{j \rightarrow \infty} \sum_{i \in \mathbb{Z}_{f}^{j}} \int_{B_{N r_{\epsilon_{j}}}^{+}} \mathcal{A} \epsilon\left(v_{j}\right) \epsilon\left(v_{j}\right) d x .
\end{aligned}
$$

Now let $w_{j}^{\prime}=\bigcup_{i \in \mathbb{Z}_{f, 1}^{j} \backslash \mathbb{Z}_{f}^{j}} Q_{i, 2}^{\epsilon_{j}}$.

For any $i \in \mathbb{Z}_{f, 1}^{j} \backslash \mathbb{Z}_{f}^{j}$ we have

$$
\begin{aligned}
& \int_{B_{N r_{\epsilon_{j}}}^{+}} \mathcal{A} \epsilon\left(v_{j}\right) \epsilon\left(v_{j}\right) d x \leq M(N) \int_{B_{N r_{\epsilon_{j}}}^{+}}\left|D v_{j}\right|^{2} d x \\
& \quad \leq \frac{1}{r_{\epsilon_{j}}^{2}} M(N) \int_{B_{N r_{\epsilon_{j}}}^{+}}|D h|^{2}\left|w_{j}\right|^{2} d x+\int_{B_{N r_{\epsilon_{j}}}^{+}}\left|D w_{j}\right|^{2} d x .
\end{aligned}
$$

Then using (4.36) and the equi-integrability and $L^{\infty}$ bound of $w_{j}$ we obtain

$$
\begin{aligned}
& \limsup _{j \rightarrow \infty} \sum_{i \in \mathbb{Z}_{f, 1}^{j} \backslash \mathbb{Z}_{f}^{j}} \cdot \int_{B_{N r_{\epsilon_{j}}}^{+}} \mathcal{A} \epsilon\left(v_{j}\right) \epsilon\left(v_{j}\right) d x \\
& \quad \leq M(N) \lim _{j \rightarrow \infty} \frac{r_{\epsilon_{j}}}{\epsilon_{j}^{2}} \lim _{j \rightarrow \infty} \mathcal{H}^{2}\left(w_{j}^{\prime}\right) \leq M(N) \cdot c \cdot \mathcal{H}^{2}\left(\partial \Gamma_{f}^{0}\right)=0 .
\end{aligned}
$$

Next summing in (4.33) for all $i \in \mathbb{Z}_{f}^{j}$ and passing to the limit when $j \rightarrow \infty$, we get

$$
\limsup _{j \rightarrow \infty} \sum_{i \in \mathbb{Z}_{f}^{j}} \int_{B_{N \epsilon_{\epsilon_{j}}}^{+}} \mathcal{A} \epsilon\left(v_{j}\right) \epsilon\left(v_{j}\right) d x \leq \lim _{j \rightarrow \infty} \frac{r_{\epsilon_{j}}}{\epsilon_{j}^{2}} \limsup _{j \rightarrow \infty} \sum_{i \in \mathbb{Z}_{f}^{j}}\left(\epsilon_{j}^{2} \phi\left(u_{j \tau}^{i}\right)+\epsilon_{j}^{2} \delta\right) .
$$

From (4.29) we have that

$$
\sum_{i \in \mathbb{Z}_{f}^{j}} \epsilon_{j}^{2} \phi\left(u_{j \tau}^{i}\right) \leq \sum_{i \in \mathbb{Z}_{f}^{j}}\left(\epsilon_{j}^{2} \phi_{N}\left(u_{j \tau}^{i}\right)+\epsilon_{j}^{2} \frac{\delta}{2}\right) \leq M \delta+\sum_{i \in \mathbb{Z}_{f}^{j}} \epsilon_{j}^{2} \phi_{N}\left(u_{j \tau}^{i}\right) .
$$

From (4.39) and Lemma 4.9, we obtain

$$
\limsup _{j \rightarrow \infty} \sum_{i \in \mathbb{Z}_{f}^{j}} \epsilon_{j}^{2} \phi\left(u_{j \tau}^{i}\right) \leq M \delta+\limsup _{j \rightarrow \infty} \int_{\Gamma_{f}^{0}} \psi_{j} d s=M \delta+\int_{\Gamma_{f}^{0}} \phi_{N}\left(u_{\tau}\right) d s .
$$

From (4.34), (4.37) and (4.38), we obtain that there exists a positive constant $M=M\left(\|u\|_{L^{\infty}}, c, M_{1}, M_{2},\left|\Gamma_{f}^{0}\right|\right)$

$$
\limsup _{j \rightarrow \infty} \sum_{i \in \mathbb{Z}_{f, 1}^{j}} \int_{B_{N r_{\epsilon_{j}}}^{+}} \mathcal{A} \epsilon\left(v_{j}\right) \epsilon\left(v_{j}\right) d x \leq c \cdot \sum_{k, l=1}^{3} \int_{\Gamma_{f}^{0}} C_{k l} u_{k} u_{l} d s+M \delta .
$$


From (4.41) and (4.28) we obtain

$$
\limsup _{j \rightarrow \infty}\left\|v_{j}\right\|_{V}^{2} \leq\|u\|_{V}^{2}+c \sum_{k, l=1}^{3} \int_{\Gamma_{f}^{0}} C_{k l} u_{k} u_{l} d s+M \delta .
$$

Because of the fact that $M$ is a constant independent of $\delta$, the statement follows taking for example $\delta \doteq \frac{\delta}{M}$ in (4.33) and (4.30). Next the boundedness assumption for $u \in L^{\infty}\left(\Omega ; \mathbb{R}^{3}\right)$ can be removed exactly by the same arguments in 2 .

Next we make the simple observation that the functional $v \rightsquigarrow \int_{\Gamma_{f}^{0}} S H\left(\left|v_{\tau}\right|\right)-f(v)$ is continuous with respect to the weak topology on $V$. This can be seen by the trace continuity and the definition of the function $H$. We can also easily observe that the limit functional does not depend on the particular subsequence $\epsilon_{j}$ and therefore by Uryson's property for the $\Gamma$-limits, using Lemma 4.10 and Lemma 4.11 and the above observations, we proved Theorem 4.1.

The cases $c=0$ and $c=\infty$ are discussed in the following remark:

REMARK 4.12. We can see that when $c=0$ the influence of the barriers disappear in the limit problem. Indeed in this case we obtain

$$
\Gamma-\lim _{\epsilon \rightarrow 0} \mathcal{W}_{\epsilon}=\mathcal{W}
$$

where $\mathcal{W}: V \rightarrow \mathbb{R}^{3}$

$$
\mathcal{W}(v)=\frac{1}{2}\|v\|_{V}^{2}+\int_{\Gamma_{f}^{0}} S H\left(\left|v_{\tau}\right|\right)-f(v) .
$$

In the other case $c=\infty$ we obtain that

$$
\Gamma-\lim _{\epsilon \rightarrow 0} \mathcal{W}_{\epsilon}=\mathcal{W}
$$

with $\mathcal{W}: V \rightarrow \mathbb{R}^{3}$ and

$$
\mathcal{W}(u)=\left\{\begin{array}{lr}
\frac{1}{2}\|u\|_{V}^{2} & \text { if } \quad u \in V_{1} \\
\infty & \text { otherwise. }
\end{array}\right.
$$

4.2. Asymptotic analysis of the spectral problem $\mathcal{E}_{\epsilon}$. Rayleigh's principle for the operator $K^{\epsilon}$ gives us

$$
\lambda_{n}^{\epsilon}=\inf _{\substack{u \in W_{\epsilon}, u \perp u_{i}^{\epsilon} \\ i=\overline{1, n-1}}} \frac{\|u\|_{V}^{2}}{\int_{\Gamma_{f}^{0}} u_{\tau}^{2}} .
$$

where $\left\{u_{i}^{\epsilon}\right\}_{i}$ form the orthonormal sequence of eigenvectors for $K^{\epsilon}$ corresponding to the sequence of eigenvalues $\left\{\lambda_{i}^{\epsilon}\right\}_{i}$. Using trace inequality and (2.3) we obtain

$$
\lambda_{n}^{\epsilon} \geq M \text { for any } n \in \mathbf{N}
$$

with $M$ not depending on $\epsilon$, and therefore $\left\{\lambda_{n}^{\epsilon}\right\}$ is uniformly bounded from below. Next we will prove that all the limit points $\lambda_{n}$ of $\left\{\lambda_{n}^{\epsilon}\right\}_{\epsilon}>0$ are finite.

LEMmA 4.13. If $\lim _{\epsilon \rightarrow 0} \frac{r_{\epsilon}}{\epsilon^{2}}<\infty$, then we have $\limsup _{\epsilon \rightarrow 0} \lambda_{n}^{\epsilon}<\infty$ for any $n \in \mathbf{N}$. 
Proof. Let $u \in V$ such that $u=\bar{u}_{1}+\bar{u}_{2}$ where $0 \neq \bar{u}_{1} \in W$ and $0 \neq \bar{u}_{2} \in V_{1}$.

Next consider the recovering sequence for $u$, i.e. $\bar{u}_{\epsilon}$ defined in the proof of Theorem 4.1. (4.27) and (4.31). We have that $\bar{u}_{\epsilon} \in V_{\epsilon}$ and $\bar{u}_{\epsilon} \rightarrow u$ weakly in $V$. Obviously from the definition of $u \in V$ we can see that there is an $\epsilon_{0}>0$ such that

$$
\bar{u}_{\epsilon} \notin V_{1} \text { and } \bar{u}_{\epsilon} \notin W_{\epsilon}
$$

for $\epsilon<\epsilon_{0}$.

Indeed if $\bar{u}_{\epsilon} \in V_{1}$ for a subsequence still denoted by $\epsilon$, with $\epsilon \rightarrow 0$, then

$$
0=\left\langle\bar{u}_{\epsilon}, \bar{u}_{1}\right\rangle_{V} \rightarrow\left\langle\bar{u}_{1}, \bar{u}_{1}\right\rangle_{V}=\left\|\bar{u}_{1}\right\|_{V}^{2}>0
$$

and therefore the contradiction. Similarly it can be seen that $\bar{u}_{\epsilon} \notin W_{\epsilon}$ for all $\epsilon<\epsilon_{0}$. From (4.42) we have that

$$
\lambda_{1}^{\epsilon} \leq \frac{\left\|P_{W^{\epsilon}} \bar{u}_{\epsilon}\right\|_{V}^{2}}{\int_{\Gamma_{f}^{0}}\left(P_{W^{\epsilon}} \bar{u}_{\epsilon}\right)_{\tau}^{2}} \leq \frac{\left\|\bar{u}_{\epsilon}\right\|_{V}^{2}}{\int_{\Gamma_{f}^{0}} \bar{u}_{\epsilon \tau}^{2}} \leq \frac{M}{\int_{\Gamma_{f}^{0}} \bar{u}_{\epsilon \tau}^{2}} .
$$

Since $\bar{u}_{\epsilon}$ is weakly convergent to $u$ and using the continuity of the trace we get

$$
\limsup _{\epsilon \rightarrow 0} \lambda_{1}^{\epsilon} \leq \frac{M}{\int_{\Gamma_{f}^{0}} \bar{u}_{1 \tau}^{2}}<\infty,
$$

where we used the orthogonal decomposition

$$
V_{\epsilon}=W_{\epsilon} \oplus V_{1}
$$

in order to obtain

$$
\int_{\Gamma_{f}^{0}}\left(P_{W_{\epsilon}} \bar{u}_{\epsilon}\right)_{\tau}^{2}=\int_{\Gamma_{f}^{0}} \bar{u}_{\epsilon \tau}^{2} .
$$

Next we will use an induction argument to prove the statement for all $n \in \mathbf{N}$. Let us assume that

$$
\limsup _{\epsilon \rightarrow 0} \lambda_{k}^{\epsilon}<\infty \quad \text { for any } k \leq n-1 \text {. }
$$

We need to prove

$$
\limsup _{\epsilon \rightarrow 0} \lambda_{n}^{\epsilon}<\infty .
$$

Let $\left\{\lambda_{n}^{\epsilon}\right\}_{\epsilon>0}$ be a subsequence of $\left\{\lambda_{n}^{\epsilon}\right\}_{\epsilon>0}$ still denoted by $\epsilon$. Then, using the induction hypothesis (4.45), the orthonormality of the associated sequence of eigenvectors and a diagonalization argument, we find a decreasing sequence $\left\{\epsilon_{j}\right\}_{j \in \mathbf{N}}$ such that $\epsilon_{j} \rightarrow 0$ and

$$
\begin{gathered}
u_{k}^{\epsilon_{j}} \stackrel{j}{\longrightarrow} u_{k} \in \mathrm{W}, \\
\lim _{j \rightarrow \infty} \lambda_{k}^{\epsilon_{j}} \doteq \lambda_{k}<\infty,
\end{gathered}
$$

for $k=\overline{1, n-1}$.

Let $u \in V, u=\bar{u}_{1}+\bar{u}_{2}$, where $0 \neq \bar{u}_{1} \in W$ and $0 \neq \bar{u}_{2} \in V_{1}$, with

$$
\bar{u}_{1} \notin \operatorname{span}\left\{u_{1}, \ldots, u_{n-1}\right\} .
$$

We can do that because $W$ has infinite dimension.

Let $\bar{u}_{\epsilon}$ be the recovering sequence defined before such that $\bar{u}_{\epsilon} \in V_{\epsilon}$ and $\bar{u}_{\epsilon} \rightarrow u$. 
From (4.42) we obtain

$$
\lambda_{n}^{\epsilon_{j}}=\inf _{\substack{u \in W_{\epsilon_{j}}, u \perp u_{i}^{\epsilon_{j}} \\ i=\overline{1, n-1}}} \frac{\|u\|_{V}^{2}}{\int_{\Gamma_{f}^{0}} u_{\tau}^{2} d \sigma}
$$

Now consider

$$
\bar{z}^{\epsilon_{j}}=\bar{u}_{\epsilon_{j}}-\sum_{i=1}^{n-1} u_{i}^{\epsilon_{j}}\left\langle\bar{u}_{\epsilon_{j}}, u_{i}^{\epsilon_{j}}\right\rangle_{V}
$$

First we can see that

$$
\left\langle\bar{z}^{\epsilon_{j}}, u_{i}^{\epsilon_{j}}\right\rangle_{V}=0 \text { for any } i=\overline{1, n-1} .
$$

Then $\bar{z}^{\epsilon_{j}} \in V_{\epsilon_{j}}$ and $\bar{z}^{\epsilon_{j}} \notin V_{1}$ for $j$ big enough.

Indeed from (4.4) we have

$$
\left\langle\bar{u}_{\epsilon_{j}}, u_{i}^{\epsilon_{j}}\right\rangle_{V}=\lambda_{i}^{\epsilon_{j}} \int_{\Gamma_{f}^{0}} u_{i \tau}^{\epsilon_{j}} \bar{u}_{\epsilon_{j} \tau} \text { for } i=\overline{1, n-1},
$$

and from the trace continuity, the definition of $\bar{u}^{\epsilon_{j}}$, 4.46) and (4.47) letting $j$ go to the $\infty$ in (4.52) and using the result in (4.50), we have

$$
\bar{z}^{\epsilon_{j}} \rightarrow \bar{z} \doteq u-\sum_{i=1}^{n-1} u_{i} \lambda_{i} \int_{\Gamma_{f}^{0}} u_{i \tau} \bar{u}_{1 \tau} .
$$

If we suppose $z_{\tau}=0$ on $\Gamma_{f}^{0}$, this is equivalent to

$$
\left(u-\sum_{i=1}^{n-1} u_{i} \lambda_{i} \int_{\Sigma_{0}} u_{i \tau} \bar{u}_{1 \tau}\right)_{\tau}=0 \text { on } \Sigma_{0},
$$

and this is equivalent to

$$
\left(\bar{u}_{1}-\sum_{i=1}^{n-1} u_{i} \lambda_{i} \int_{\Gamma_{f}^{0}} u_{i \tau} \bar{u}_{1 \tau}\right)_{\tau}=0 \text { on } \Sigma_{0},
$$

which implies

$$
\bar{u}_{1}-\sum_{i=1}^{n-1} u_{i} \lambda_{i} \int_{\Gamma_{f}^{0}} u_{i \tau} \bar{u}_{1 \tau}=0
$$

because $\bar{u}_{1}-\sum_{i=1}^{n-1} u_{i} \lambda_{i} \int_{\Gamma_{f}^{0}} u_{i \tau} \bar{u}_{1 \tau} \in W$ and $W \perp V_{1}$.

But (4.53) leads to a contradiction with (4.48). Therefore $\bar{z}_{\tau} \neq 0$ and this implies the statement, i.e. $\bar{z}^{\epsilon_{j}} \notin V_{1}$ for $j$ big enough.

Next using (4.51) and (4.49) we obtain

$$
\lambda_{n}^{\epsilon_{j}} \leq \frac{\left\|P_{W^{\epsilon_{j}}} \bar{z}^{\epsilon_{j}}\right\|_{V}^{2}}{\int_{\Gamma_{f}^{0}}\left(P_{W^{\epsilon_{j}}} \bar{z}^{\epsilon_{j}}\right)_{\tau}^{2}} \leq \frac{\left\|z^{\epsilon_{j}}\right\|_{V}^{2}}{\int_{\Gamma_{f}^{0}}\left(\bar{z}^{\epsilon_{j}}\right)_{\tau}^{2}} \leq \frac{M}{\int_{\Gamma_{f}^{0}}\left(\bar{z}^{\epsilon_{j}}\right)_{\tau}^{2}} .
$$

Passing to the limit when $j \rightarrow \infty$ we obtain

$$
\limsup _{j \rightarrow \infty} \lambda_{n}^{\epsilon_{j}} \leq \frac{M}{\int_{\Gamma_{f}^{0}} \bar{z}_{\tau}^{2}}<\infty .
$$


So we have proved that any subsequence of $\lambda_{n}^{\epsilon}$ has a subsequence $\left\{\lambda_{n}^{\epsilon_{j}}\right\}_{j \in \mathbf{N}}$ such that (4.54) is satisfied. Therefore we have that

$$
\limsup _{\epsilon \rightarrow 0} \lambda_{n}^{\epsilon}<\infty
$$

for any $n \in \mathbf{N}$.

The next corollary shows that the weak-limits $u_{n}$ of the sequence $\left\{u_{n}^{\epsilon}\right\}_{\epsilon>0}$ of the normal eigenvectors associated to the eigenvalue $\lambda_{n}^{\epsilon}$ cannot be zero.

Corollary 4.14. Let $\left\{u_{n}^{\epsilon}\right\}_{n \in \mathbf{N}}$ be the orthonormal sequence of eigenvectors associated to $\lambda_{n}^{\epsilon}$ for the problem $\left(\mathcal{E}_{\epsilon}\right)$. Then for any $n \in \mathbb{N}$ we have that every weak-limit $u_{n}$ of $\left\{u_{n}^{\epsilon}\right\}_{n \in \mathbf{N}}$ (i.e. $u_{n}$ such that on a subsequence $u_{n}^{\epsilon} \stackrel{\epsilon}{\rightarrow} u_{n}$ ) is nonzero.

Proof. Let $n \in \mathbb{N}$ be arbitrarily fixed. Let $u_{n}$ be a weak limit of $\left\{u_{n}^{\epsilon}\right\}$. Thus there exists a subsequence of $\left\{u_{n}^{\epsilon}\right\}_{\epsilon}$ still denoted by $\epsilon$ such that $u_{n}^{\epsilon} \rightarrow u_{n}$.

Using the variational form of $\mathcal{E}_{\epsilon}$ and the normality of $\left\{u_{n}^{\epsilon}\right\}$ we have

$$
\lambda_{n}^{\epsilon}=\frac{1}{\int_{\Gamma_{f}^{0}} u_{n \tau}^{\epsilon}{ }^{2}} .
$$

Letting $\epsilon$ go to zero above we obtain

$$
\lambda_{n}=\frac{1}{\int_{\Gamma_{f}^{0}} u_{n \tau}^{2}} .
$$

Next using Lemma 4.13 we obtain that

$$
\int_{\Gamma_{f}^{0}} u_{n \tau}^{2} \neq 0
$$

and this together with the arbitrariness of $n$ implies the statement.

Let us now consider the duality operator $J^{\epsilon}: V_{\epsilon} \rightarrow\left(V_{\epsilon}\right)^{\prime}$

$$
\left\langle J^{\epsilon} u, w\right\rangle_{\left(V_{\epsilon}\right)^{\prime}, V_{\epsilon}}=\langle u, w\rangle_{V} \quad \text { for any } u, w \in V_{\epsilon} .
$$

$J^{\epsilon}$ is an operator of subdifferential type

$$
\begin{gathered}
J^{\epsilon}=\partial \varphi^{\epsilon}, \quad \varphi^{\epsilon}: V^{\epsilon} \rightarrow \mathbb{R}, \\
\varphi^{\epsilon}(u)=\frac{1}{2}\|u\|_{V}^{2} .
\end{gathered}
$$

Lemma 4.15. The sequence of operators $J^{\epsilon}$ is $G$ convergent to $\partial \varphi$, with respect to the weak $\times$ strong topology in $V \times V^{\prime}$.

Proof. From the proof of Theorem 4.1 the sequence of functionals $\left\{\varphi^{\epsilon}\right\}$ is $\Gamma$-convergent weakly in $V$ to $\varphi$ given by

$$
\varphi(v)=\frac{1}{2}\|v\|_{V}^{2}+\frac{1}{2} c \int_{\Gamma_{f}^{0}} C_{i j} v_{i} v_{j}=\frac{1}{2}\|v\|_{V}^{2}+\frac{1}{2} c \int_{\Gamma_{f}^{0}} v_{\tau} C v_{\tau},
$$

where $c$ and the matrix $\left(C_{i j}\right)_{i, j=1,3}$ are defined in Theorem 4.1. Using the $G$-convergence result for subdifferentials of $\Gamma$-convergent sequences (see Attouch [5, Th. 3.67]), we have 
that the $\Gamma$-convergences of the sequence $\varphi^{\epsilon}$ to $\varphi$ imply the $G$-convergence of the subdifferentials,

$$
\partial \varphi^{\epsilon} \stackrel{G}{\rightarrow} \partial \varphi
$$

TheOREM 4.16. There is a decreasing sequence $\left\{\epsilon_{j}\right\}_{j} \in \mathbf{N}$ with $\epsilon_{j} \rightarrow 0$ such that $u_{n}^{\epsilon_{j}} \rightarrow u_{n}, \quad \lambda_{n}^{\epsilon_{j}} \rightarrow \lambda_{n}$ where $\left(\lambda_{n}, u_{n}\right)$ solves the limit problem, $\lambda_{n} \in \mathbb{R}$ and $u_{n} \in W$ such that

$$
\begin{gathered}
\sigma\left(u_{n}\right)=\mathcal{A} \epsilon\left(u_{n}\right), \quad \operatorname{div} \sigma\left(u_{n}\right)=0, \quad \text { in } \Omega, \\
u_{n}=0 \text { on } \Gamma_{d} \quad \sigma_{33}\left(u_{n}\right)=0 \text { on } \Sigma_{0}, \\
\sigma_{\tau}\left(u_{n}\right)=u_{n \tau}\left(\lambda_{n} I_{3}-c C\right) \quad \text { on } \quad \Gamma_{f}^{0},
\end{gathered}
$$

where $I_{3}$ is the unity matrix in $\mathcal{M}^{3 \times 3}$ and $c$ and the matrix $C$ have been defined in Theorem 4.1 .

Proof. Let an arbitrary fixed $n \in \mathbf{N}$. Let $\left\{\lambda_{n}^{\epsilon}\right\}_{\epsilon>0}$ be the sequence of eigenvalues for the problem $\left(\mathcal{E}_{\epsilon}\right)$ and $u_{n}^{\epsilon}$ the corresponding orthonormal sequence of eigenvectors. Then there is a subsequence $\left\{\epsilon_{j}\right\}_{j} \in \mathbf{N}$ such that

$$
u_{n}^{\epsilon_{j}} \rightarrow u_{n} \text { and } \lambda_{n}^{\epsilon_{j}} \rightarrow \lambda_{n} .
$$

We have proved in Lemma 4.13 that $\lambda_{n}<\infty$ for all $n \in \mathbb{N}$.

Let $f_{n}^{\epsilon_{j}} \in V^{\prime}$ be defined as

$$
f_{n}^{\epsilon_{j}}(w)=\lambda_{n}^{\epsilon_{j}} \int_{\Gamma_{f}^{0}} u_{n \tau}^{\epsilon_{j}} w_{\tau} \quad \text { for all } w \in V .
$$

Using the variational formulation (4.4) we have

$$
f_{n}^{\epsilon_{j}}(w)=\left\langle J^{\epsilon_{j}} u_{n}^{\epsilon_{j}}, w\right\rangle_{\left(V_{\epsilon_{j}}\right)^{\prime}, V_{\epsilon_{j}}} \text { for all } w \in V_{\epsilon_{j}} .
$$

This implies

$$
f_{n}^{\epsilon_{j}} \in \partial \varphi^{\epsilon_{j}}\left(u_{n}^{\epsilon_{j}}\right)
$$

The next observation is that

$$
f_{n}^{\epsilon_{j}} \stackrel{j \rightarrow \infty}{\longrightarrow} f_{n} \quad \text { strongly in } V^{\prime}
$$

where

$$
f_{n}(w)=\lambda_{n} \int_{\Gamma_{f}^{0}} u_{n \tau} w_{\tau} \quad \text { for all } w \in V .
$$

The proof of the above convergence is straightforward. Indeed,

$$
\left\|f_{n}^{\epsilon_{j}}-f_{n}\right\|_{V^{\prime}}=\sup _{\substack{w \in V \\\|w\|_{V} \leq 1}}\left|\lambda_{n}^{\epsilon_{j}} \int_{\Gamma_{f}^{0}} u_{n \tau}^{\epsilon_{j}} w_{\tau}-\lambda_{n} \int_{\Gamma_{f}^{0}} u_{n \tau} w_{\tau}\right| .
$$


Now from the reflexivity of the space $V$ we have that there exists $w_{0}^{j} \in V$ with $\left\|w_{0}^{j}\right\|_{V} \leq 1$ such that

$$
\begin{aligned}
& \left\|f_{n}^{\epsilon_{j}}-f_{n}\right\|_{V^{\prime}}=\left|\lambda_{n}^{\epsilon_{j}} \int_{\Gamma_{f}^{0}} u_{n \tau}^{\epsilon_{j}} w_{0 \tau}^{j}-\lambda_{n} \int_{\Gamma_{f}^{0}} u_{n \tau} w_{0 \tau}^{j}\right| \\
& =\left|\left(\lambda_{n}^{\epsilon_{j}}-\lambda_{n}\right) \int_{\Gamma_{f}^{0}} u_{n \tau}^{\epsilon_{j}} w_{0 \tau}^{j}+\lambda_{n} \int_{\Gamma_{f}^{0}}\left(u_{n \tau}^{\epsilon_{j}}-u_{n \tau}\right) w_{0 \tau}^{j}\right| .
\end{aligned}
$$

Thus, from Cauchy-Schwartz inequality

$$
\begin{gathered}
\left\|f_{n}^{\epsilon_{j}}-f_{n}\right\|_{V^{\prime}} \leq\left|\lambda_{n}^{\epsilon_{j}}-\lambda_{n}\right|\left(\int_{\Gamma_{f}^{0}}\left|u_{n \tau}^{\epsilon_{j}}\right|^{2}\right)^{1 / 2}\left(\int_{\Gamma_{f}^{0}}\left|w_{0 \tau}^{j}\right|^{2}\right)^{1 / 2} \\
+\left|\lambda_{n}\right|\left(\int_{\Gamma_{f}^{0}}\left|u_{n \tau}^{\epsilon_{j}}-u_{n \tau}\right|^{2}\right)^{1 / 2}\left(\int_{\Gamma_{f}^{0}}\left|w_{0 \tau}^{j}\right|^{2}\right)^{1 / 2} \cdot
\end{gathered}
$$

Next we will use the following interpolation inequality (see [22]):

Lemma 4.17. Let $\Omega \subset \mathbf{R}^{d}$ be as above and let $\alpha \in\left[2, \frac{2(d-1)}{d-2}\right]$ if $d \geq 3$ and $\alpha \geq 2$ if $d=2$. Then, for $\beta=\frac{d(\alpha-2)+2}{2 \alpha}$ if $d \geq 3$ or if $d=2$ and $\alpha=2$, and for all $\left.\beta \in\right] \frac{\alpha-1}{\alpha}, 1[$ if $d=2$ and $\alpha>2$, there exists a constant $C=C(\beta)$ such that

$$
\|v\|_{L^{\alpha}(\partial \Omega)} \leq C\|v\|_{L^{2}(\Omega)}^{1-\beta}\|v\|_{H^{1}(\Omega)}^{\beta}, \quad \forall v \in H^{1}(\Omega) .
$$

In our case $d=3, \alpha=2$ and $\beta=\frac{1}{2}$, and thus the inequality becomes

$$
\left\|u_{\tau}\right\|_{L^{2}(\Sigma)}^{2} \leq M\|u\|_{H^{1}(\Omega)}\|u\|_{L^{2}(\Omega)}, \forall u \in V .
$$

Using the trace inequality, (4.63) and the fact that $\left\|w_{0}^{j}\right\|_{V} \leq 1$, we obtain

$$
f_{n}^{\epsilon_{j}} \stackrel{j \rightarrow \infty}{\longrightarrow} f_{n} \text { strongly in } V^{\prime} .
$$

Therefore from (4.60), (4.61) and using the Lemma 4.15 we obtain that

$$
f_{n} \in \partial \varphi\left(u_{n}\right) \text {. }
$$

But (4.64) is equivalent with

$$
\left\langle u_{n}, w\right\rangle_{V}=\int_{\Gamma_{f}^{0}} u_{n \tau}\left(\lambda_{n} I_{3}-c C\right) w_{\tau} \text { for any } w \in W,
$$

which is the variational formulation for the problem (4.57), (4.58), (4.59).

From the arbitrariness of $n \in \mathbf{N}$ we have that the Theorem 4.16 is proved for all positive integers $n$.

The main homogenization result of this section is:

Theorem 4.18. If $c=\lim _{\epsilon \rightarrow 0} \frac{r_{\epsilon}}{\epsilon^{2}}<\infty$, then for any $n \in \mathbb{N}$ we have:

i) $\lim _{\epsilon \rightarrow 0} \lambda_{n}^{\epsilon}=\lambda_{n}$ on the entire sequence, and $\lambda_{n}$ is the $\mathrm{n}$-th eigenvalue of the limit problem.

ii) There is a decreasing sequence $\left\{\epsilon_{j}\right\}_{j \in \mathbf{N}}$ with $\epsilon_{j} \rightarrow 0$ such that $u_{n}^{\epsilon_{j}}-u_{n}$, where $u_{n}$ is the normal eigenvector for the limit problem associated to $\lambda_{n}$. 
Proof. Suppose there is a $\lambda$ eigenvalue of the limit problem such that $\lambda \neq \lambda_{n}$ for any $n \in \mathbf{N}$.

Let $u \in W$ be the normal eigenvector associated to $\lambda$, i.e., $\|u\|_{V}=1$ and

$$
\langle u, w\rangle_{V}=\int_{\Gamma_{f}^{0}} u_{\tau}\left(\lambda I_{3}-c C\right) w_{\tau} \text { for any } w \in W .
$$

Now obviously there is $m \in \mathbf{N}$ such that

$$
\lambda<\lambda_{m+1}
$$

From the Lax-Milgram lemma we have that there exists $w^{\epsilon} \in W^{\epsilon}$ such that

$$
\left\langle J^{\epsilon} w^{\epsilon}, w\right\rangle_{\left(V_{\epsilon}{ }^{\prime}, V_{\epsilon}\right)}=\lambda \int_{\Gamma_{f}^{0}} u_{\tau} w_{\tau} \quad \text { for all } w \in W_{\epsilon} .
$$

It can easily be seen that $w^{\epsilon}$ is bounded in the norm of $V$. Then on a subsequence still denoted by $\epsilon$ we have

$$
w^{\epsilon} \rightarrow \bar{w} \text { as } \epsilon \rightarrow 0
$$

for some $\bar{w} \in W$. But if we consider $f_{\lambda} \in V^{\prime}$ with $f_{\lambda}(w)=\lambda \int_{\Gamma_{f}^{0}} u_{\tau} w_{\tau}$, then clearly from the definition of $w_{\epsilon}$ an $J^{\epsilon}$ we have

$$
f_{\lambda}(w)=\left\langle J^{\epsilon} w^{\epsilon}, w\right\rangle_{\left(V_{\epsilon}{ }^{\prime}, V_{\epsilon}\right)} \Longrightarrow f_{\lambda} \in \partial \varphi^{\epsilon}\left(w^{\epsilon}\right) .
$$

So using the $G$-convergence result stated in Lemma 4.15 we obtain

$$
f_{\lambda} \in \partial \varphi(\bar{w}) \Longleftrightarrow\langle\bar{w}, v\rangle_{V}+c \int_{\Gamma_{f}^{0}} v_{\tau} C \bar{w}_{\tau}=\lambda \int_{\Gamma_{f}^{0}} u_{\tau} v_{\tau}
$$

for any $v \in W$.

Therefore,from (4.66) we have that $u=\bar{w}$. Now by Uryson's property we can see that

$$
w^{\epsilon} \rightarrow u \text { when } \epsilon \rightarrow 0 .
$$

Let

$$
v^{\epsilon}=w^{\epsilon}-\sum_{i=1}^{m} u_{i}^{\epsilon}\left\langle w^{\epsilon}, u_{i}^{\epsilon}\right\rangle_{V} .
$$

Using the interpolation inequality (4.63) and (4.4) we obtain

$$
\left\langle w^{\epsilon}, u_{i}^{\epsilon}\right\rangle_{V}=\lambda_{i}^{\epsilon} \int_{\Gamma_{f}^{0}} u_{i \tau}^{\epsilon} w_{\tau}^{\epsilon} \stackrel{\epsilon}{\longrightarrow} \lambda_{i} \int_{\Gamma_{f}^{0}} u_{i \tau} u_{\tau} \quad \text { for } i=\overline{1, m}
$$

On the other hand, using the definition of $w^{\epsilon}$ we can see that

$$
\left\langle w^{\epsilon}, u_{i}^{\epsilon}\right\rangle_{V}=\lambda \int_{\Gamma_{f}^{0}} u_{\tau} u_{i \tau}^{\epsilon} \stackrel{\epsilon}{\longrightarrow} \lambda \int_{\Gamma_{f}^{0}} u_{i \tau} u_{\tau} \quad \text { for } i=\overline{1, m} .
$$

Now because $\lambda \neq \lambda_{i}$ for all $i=\overline{1, m}$ from the last two relations, we have that

$$
\int_{\Gamma_{f}^{0}} u_{i \tau} u_{\tau}=0 \text { for all } i=\overline{1, m} .
$$


Thus $\left\langle w^{\epsilon}, u_{i}^{\epsilon}\right\rangle_{V} \stackrel{\epsilon}{\longrightarrow} 0$ and therefore $v^{\epsilon} \rightarrow u$ weakly in $V$. Noting that $v^{\epsilon} \in W^{\epsilon}$ and $v^{\epsilon} \perp u_{i}^{\epsilon}$ for all $i=\overline{1, m}$ from Rayleigh's principle for $\left(\mathcal{E}_{\epsilon}\right)$, we have

$$
\lambda_{m+1}^{\epsilon} \leq \frac{\left\|v^{\epsilon}\right\|_{V}^{2}}{\int_{\Gamma_{f}^{0}}\left(v_{\tau}^{\epsilon}\right)^{2}}
$$

Now, from the definition of $w^{\epsilon}$ and the trace continuity we have

$$
\lim _{\epsilon \rightarrow 0}\left\|v^{\epsilon}\right\|_{V}^{2}=\lim _{\epsilon \rightarrow 0}\left\|w^{\epsilon}\right\|_{V}^{2}=\lambda \int_{\Gamma_{f}^{0}}\left(u_{\tau}\right)^{2} .
$$

From the last relation, the inequality (4.63) and Theorem 4.16, passing to the limit when $\epsilon \rightarrow 0$ in (4.68), we obtain the contradiction. So i) has been proved and ii) is exactly the same as in Theorem 4.16

Next, following an idea in [5], we give a Mosco-convergence (see [5] for the definition of Mosco-convergence) result for the case $c=\lim _{\epsilon \rightarrow 0} \frac{r_{\epsilon}}{\epsilon^{2}}<\infty$ :

Theorem 4.19. Let $c=\lim _{\epsilon \rightarrow 0} \frac{r_{\epsilon}}{\epsilon^{2}}<\infty$ and $i \in \mathbf{N}$ arbitrary fixed and let $\left\{\lambda_{n}^{\epsilon}, u_{n}^{\epsilon}\right\}_{n}$ be the couple of eigenvalues and normal eigenfunctions for $\mathcal{E}_{\epsilon}$.

Then if $m_{i}$ is the order of multiplicity of $\lambda_{i}$, i.e.

$$
\lambda_{i-1}<\lambda_{i}=\lambda_{i+1}=\ldots=\lambda_{i+m_{i}-1}<\lambda_{i+m_{i}},
$$

then the sequence of subspaces generated by $\left\{u_{i}^{\epsilon}, \ldots, u_{i+m_{i}-1}^{\epsilon}\right\}$ Mosco-converge in $L^{2}(\Omega)$ to the eigenspace $\left\{\tilde{u}_{i}, \ldots, \tilde{u}_{i+m_{i}-1}\right\}$ associated to $\lambda_{i}$.

Proof. We remark that the multiplicity of $\lambda_{i}^{\epsilon}$ might be strictly smaller than that of $\lambda_{i}$. So if we denote

$$
\operatorname{span}\left\{u_{i}^{\epsilon}, \ldots, u_{i+m_{i}-1}^{\epsilon}\right\} \doteq S_{i}^{\epsilon} \text { and } \operatorname{span}\left\{\tilde{u}_{i}, \ldots, \tilde{u}_{i+m_{i}-1}\right\} \doteq S_{i},
$$

we can see that as in the above remark $S_{i}^{\epsilon}$ may be strictly larger than the eigenspace of $\lambda_{i}^{\epsilon}$. Now from Theorem 4.18 we have that, for any $n \in \mathbb{N}$, there is a subsequence still denoted by $\epsilon$ such that

$$
\lim _{\epsilon \rightarrow 0} \lambda_{n}^{\epsilon}=\lambda_{n} \text { and } u_{n}^{\epsilon_{j}} \rightarrow u_{n} \quad \text { weakly in } V,
$$

where $\left(u_{n}, \lambda_{n}\right)$ solve the spectral limit problem (4.57), (4.58) and (4.59).

From the linearity of $\mathcal{E}_{\epsilon}$ and $\mathcal{E}$ we can say that

$$
\limsup _{\epsilon \rightarrow 0} S_{i}^{\epsilon} \subset S_{i}
$$

We can easily see that for arbitrary fixed $l, j \in\left\{i, \ldots, i+m_{i}-1\right\}$, with $l \neq j$ and

$$
u_{l}^{\epsilon} \rightarrow u_{l} \text { and } u_{j}^{\epsilon} \rightarrow u_{j},
$$

we have

$$
u_{l} \neq u_{j}
$$

Indeed suppose that there are $l, j \in\left\{i, \ldots, i+m_{i}-1\right\}$, with $l \neq j$ such that $u_{l}=u_{j}$. Then from

$$
2=\left\|u_{l}^{\epsilon}-u_{j}^{\epsilon}\right\|_{V}^{2}=\int_{\Sigma_{0}}\left(\lambda_{l}^{\epsilon} u_{l \tau}^{\epsilon}-\lambda_{j}^{\epsilon} u_{j \tau}^{\epsilon}\right)\left(u_{l \tau}^{\epsilon}-u_{j \tau}^{\epsilon}\right)
$$

passing to the limit when $\epsilon \rightarrow 0$, using the inequality (4.63) we obtain the contradiction. 
Next we will prove that set $\left\{u_{i}, \ldots, u_{i+m_{i}-1}\right\}$ is linear independent. Indeed let

$$
\sum_{k=i}^{i+m_{i}-1} c_{k} u_{k}=0
$$

We have for any $j \in\left\{i, \ldots, i+m_{i}-1\right\}$ that

$c_{j}=\left\langle\sum_{k=i}^{i+m_{i}-1} c_{k} u_{k}^{\epsilon}, u_{j}^{\epsilon}\right\rangle_{V}=\lambda_{j}^{\epsilon} \int_{\Sigma_{0}}\left(\sum_{k=i}^{i+m_{i}-1} c_{k} u_{k \tau}^{\epsilon}\right) u_{j \tau}^{\epsilon} \stackrel{\epsilon}{\longrightarrow} \lambda_{j} \int_{\Sigma_{0}}\left(\sum_{k=i}^{i+m_{i}-1} c_{k} u_{k \tau}\right) u_{j \tau}=0$, where the last equality above comes from (4.71).

Using the linear independence of $\left\{u_{i}, \ldots, u_{i+m_{i}-1}\right\}$, (4.70) and the fact that the dimension of the eigenspace associated to $\lambda_{i}$ is $m_{i}$, we have in fact that

$$
S_{i}=\operatorname{span}\left\{u_{i}, \ldots, u_{i+m_{i}-1}\right\}
$$

and therefore

$$
\limsup _{\epsilon \rightarrow 0} S_{i}^{\epsilon}=S_{i}
$$

Because of the compact imbedding of $V$ in $\left[L^{2}\right]^{3}$ we have that there is a subsequence $\epsilon_{j}$ such that

$$
\liminf _{\epsilon \rightarrow 0} S_{i}^{\epsilon}=\limsup _{j \rightarrow \infty} S_{i}^{\epsilon_{j}}
$$

Now if there is $v$ such that

$$
v \notin \liminf _{\epsilon \rightarrow 0} S_{i}^{\epsilon},
$$

then from the above relation we have

$$
v \notin \limsup _{j \rightarrow \infty} S_{i}^{\epsilon_{j}}=S_{i}
$$

which implies

$$
S_{i} \subset \liminf _{\epsilon \rightarrow 0} S_{i}^{\epsilon}
$$

So we have proved the statement.

In the next remark we will briefly discuss the cases $c=0$ and $c=\infty$.

REMARK 4.20. The case $c=0$ can be seen as a particular case of the previous theorems. The limit problem for the problem $\mathcal{E}_{\epsilon}$ is

$$
\begin{gathered}
\sigma\left(u_{n}\right)=\mathcal{A} \epsilon\left(u_{n}\right), \quad \operatorname{div} \sigma\left(u_{n}\right)=0, \quad \text { in } \Omega, \\
u_{n}=0 \text { on } \Gamma_{d} \quad \sigma_{33}\left(u_{n}\right)=0 \text { on } \Sigma_{0}, \\
\sigma_{\tau}\left(u_{n}\right)=\lambda_{n} u_{n \tau} \quad \text { on } \quad \Gamma_{f}^{0} .
\end{gathered}
$$

In the other case $c=\infty$ we have seen that the sequence $\left\{\varphi^{\epsilon}\right\}_{\epsilon>0}$ defined in Lemma $4.15 \Gamma$-converges to $\varphi$, and we have

$$
\varphi(u)= \begin{cases}\|u\|_{V}^{2} d x & \text { if } u \in V_{1}, \\ \infty & \text { otherwise. }\end{cases}
$$

Now suppose that there is $n \in \mathbf{N}$ such that $\lambda_{n}^{\epsilon} \stackrel{\epsilon}{\rightarrow} \lambda_{n}<\infty$.

Now using the same approach as before, from Theorem 4.16 and Lemma 4.15 we obtain that $f_{\lambda} \in \partial \varphi\left(u_{n}\right)$, where $f_{\lambda}$ has been defined above. This means that

$$
u_{n} \in \operatorname{Dom}(\varphi)=V_{1} \text {. }
$$


But we know that $u_{n}^{\epsilon} \in W^{\epsilon} \subset W$ which means that

$$
u_{n} \in W .
$$

Using the fact that $W=V_{1}{ }^{\perp}$ in $V$ we obtain $u_{n}=0$, which contradicts Corollary 4.14. Then our assumption that $\lambda_{n}<\infty$ is false. Now from the variational form of (4.4), if $u_{n}^{\epsilon}$ is the normal eigenvector associated to $\lambda_{n}^{\epsilon}$, we have

$$
\frac{1}{\lambda_{n}^{\epsilon}}=\int_{\Sigma_{0}}\left(u_{n \tau}^{\epsilon}\right)^{2} .
$$

Consider $u_{n} \in W$ to be the weak limit of $u_{n}^{\epsilon}$ when $\epsilon \rightarrow 0$. Passing to the limit for $\epsilon \rightarrow 0$ in the equality above we obtain

$$
\int_{\Sigma_{0}}\left(u_{n \tau}\right)^{2}=0
$$

This together with the fact that $u_{n} \in W$ and $W \perp V_{1}$ give us that $u_{n}=0$. So in this case we have that all the eigenvectors of the $\mathcal{E}_{\epsilon}$ converges to zero and all the eigenvalues of the same problem converges to $\infty$.

5. Physical interpretation. Here we give the physical interpretation of the previous theoretical results concerning the macroscopic behavior of a fault with small-scale heterogeneity of rupture resistance (small-scale barriers). Through Theorems 4.1 and 4.16 we have obtained an effective (or equivalent) friction law which, used on a homogeneous fault, leads to a slip evolution similar to the one produced on the heterogeneous fault. More precisely, for a fault which has $\epsilon$-periodically distributed barriers of radius $r_{\epsilon}$, we have proved that for $0<c=: \lim _{\epsilon \rightarrow 0} r_{\epsilon} / \epsilon^{2}<\infty$ the sequence of energy functionals $\Gamma$-converges to a limit energy functional. This limit functional is associated to another slip-weakening friction problem called the equivalent friction law. These results can be interpreted in the context of a barrier erosion process during the nucleation phase of an earthquake.

The earthquake nucleation (or initiation) phase, preceding the dynamic rupture, has been pointed out by detailed seismological observations (e.g. 21, 19]), and it has been recognized in laboratory experiments (e.g. [18, 29]) to be related to the slip-weakening friction. This physical model was thereafter used in the qualitative description of the initiation phase in unbounded (e.g. 9, 1]) and bounded (e.g. [16, 34]) fault models. Important physical properties of the nucleation phase (characteristic time, critical fault length, etc.) were obtained in [9, 16, 17] through simple mathematical properties of the unstable evolution.

During the nucleation phase, the stress concentration at the boundary between the barriers and the slipping zone exceeds the barriers' strength, and a part of the barrier is broken (i.e. it is transformed in a slipping zone). The evolution of the shape and of the distribution of the barriers can change the effective frictional properties of the fault and can explain the qualitatively different behaviors with the same local friction law.

In order to see how the barriers' evolution changes the effective friction properties during the initiation phase, let us imagine that we deal with an external loading process on the time interval $[0, T]$. Since the loading rate of the tectonic plates is very slow we 
can suppose that the process is quasi-static. In this context $[0, T]$, the nucleation (or initiation) phase of an earthquake turns out to be the transition between the quasi-static and the dynamic slip. The fault is supposed to have periodically distributed barriers of period $\epsilon$ (small non-dimensional distance with respect to the fault length) and of a variable diameter $r_{\epsilon}(t)$ (non-dimensional length) with $t \in[0, T]$. The erosion of the barriers is described by the fact that the function $t \rightarrow r_{\epsilon}(t)$ is non-increasing. Regarding the evolution of the parameter

$$
A_{\epsilon}(t)=: \frac{r_{\epsilon}(t)}{\epsilon^{2}}
$$

we can distinguish three periods of time. At the beginning of the process, $\left[0, T_{1}\right]$, the diameter of the barriers is large (i.e. $A_{\epsilon}(t)$ is very large). In the second period of time $\left[T_{1}, T_{2}\right]$ the parameter $A_{\epsilon}(t)$ is of the order of unity and in the last period $\left[T_{2}, T\right]$ the parameter $A_{\epsilon}(t)$ is very small.

1) In the first period of time $\left[0, T_{1}\right]$ the barriers are too large with respect to the distance between them (i.e. $c(t)=: \lim _{\epsilon \rightarrow 0} r_{\epsilon}(t) / \epsilon^{2}=\infty$ ), and the equivalent fault is locked (i.e. no large-scale slip even if we can have a small-scale slip). This means that the presence of the "large" barriers (i.e. with diameters of the same order of the distance between them) will imply that the effective static friction force is larger than the local one. Such a fault can stand "large-scale" locked without slipping even if the loading is greater than the local friction resistance.

2) In the second period of time $\left[T_{1}, T_{2}\right]$ the ratio between the barrier radius and the inter-barrier distance is of order of the ratio between the the inter-barrier distance and the fault length (i.e. $0<c(t)=: \lim _{\epsilon \rightarrow 0} r_{\epsilon}(t) / \epsilon^{2}<\infty$ ). In this case on the equivalent fault is acting a slip-weakening friction law with a smaller weakening rate. That means that during this period of time the equivalent fault has a larger critical slip $D_{c}$. The presence of barriers that slow down the growth of the instability is accounted for in the effective law by an initial weakening rate that is much smaller than that for the local laws. Since the initial weakening of a friction law determines the initiation duration, as discussed in 23], the initiation time associated with a large earthquake which develops on a large area of a heterogeneous fault can be important. The equivalent slip-weakening rate may be also negative, hence a slip-hardening effect can be expected. These types of friction properties were used in 35] in describing the dynamic rupture arrest. Moreover, the large-scale (equivalent) friction law is not isotropic (i.e. the tangential stress and the slip are not collinear). This can be explained by the fact that the periodic distribution of the barriers is not isotropic, hence the limit problem will heritage this anisotropic geometrical perturbation.

3) In the third period of time $\left[T_{2}, T\right]$ the barriers are too small with respect to the distance between them (i.e. $c(t)=: \lim _{\epsilon \rightarrow 0} r_{\epsilon}(t) / \epsilon^{2}=0$ ), and the presence of the barriers does not affect the friction law on the equivalent fault. That means that the effective friction law is the same as the local one only in the last stage of nucleation phase. Moreover the slip weakening rate at the end of the initiation is larger than the rate of the initial stage of nucleation.

Let us now summarize the role played by the process of erosion of the barriers in the effective properties of the homogenized fault. In this context the time period $\left[0, T_{1}\right]$ 
turns to be the "(effective) locking period", the second one, $\left[T_{1}, T_{2}\right]$, is the "first stage of (effective) initiation" and the last one, $\left[T_{2}, T\right]$, becomes the "last stage of (effective) initiation".

i) The effective friction resistance (static friction) is greater than the local one.

ii) The slip-weakening rate is smaller at the beginning of initiation phase than at the end. This implies a concave shape of the friction distribution with respect to the slip of the effective friction law. From the concavity of the friction law we can expect a long initiation phase.

iii )A negative weakening rate (i.e. hardening of the friction force) can be present in some cases at the beginning of the initiation phase.

iv) A loss of the isotropicity of the friction force can be noted during the first stage of the nucleation phase.

We have to mention that the partition of the initiation phase into two stages with two weakening rates was also pointed out in [10] into a different context. Indeed, in [10] they analyze a dynamic two-dimensional (anti-plane) process, and the separation between the two stages is given by the fact that barriers are (almost) instantaneously broken. In contrast to the present analysis this separation is given by a quasi-static erosion of the barriers.

\section{REFERENCES}

[1] J.-P. Ampuero, J.-P. Vilotte and F.J. Sanchez-Sesma, Nucleation of rupture under slip dependent friction law: simple models of fault zone, J. Geophys. Res., vol 107, B12, 101029/2001JB000452 2002.

[2] N. Ansini, The nonlinear sieve problem and applications to thin films, Asymptotic Anal., vol. 39, 2, 2004, pp. 113-145. MR2093896 (2005f:35019)

[3] N. Ansini and A. Braides, Asymptotic analysis of periodically-perforated nonlinear media, J. Math. Pures Appl., vol. 81, 2002, pp. 439-451. MR1907765 (2003f:35015)

[4] H. Aochi and E. Fukuyama, Three-dimensional nonplanar simulation of the 1991 Landers earthquake, J. Geophys. Res., vol. 107, 2001, 10.1028/2000JB000032.

[5] H. Attouch, Variational Convergence for Functions and Operators, Pitman, Boston, 1984. MR:0773850 (86f:49002)

[6] A. Braides and A. Defranceschi, Homogenization of Multiple Integrals, Oxford University Press, Oxford, 1998. MR1684713 (2000g:49014)

[7] A. Braides, A. Defranceschi and E. Vitali, Homogenization of free discontinuity problems, Arch. Rational Mech. Anal., vol. 135, 1996, pp. 297-356. MR1423000 (98j:73009)

[8] A. Brillard, M. Lobo and E. Perez, Homogénisation par épiconvergence en élasticité linéaire, Math. Modelling and Num. Analysis, vol. 24, 1, 1990, pp. 5-26. MR.1034896 (91b:73007)

[9] M. Campillo and I. R. Ionescu, Initiation of antiplane shear instability under slip dependent friction, J. of Geophys. Res., vol. 122, B9, 1997, pp. 20363-20371.

[10] M. Campillo, P. Favreau, I.R. Ionescu and C. Voisin, On the effective friction law of an heterogeneous fault, J. Geophys. Res., vol. 106, B8, 2001, pp. 16307-16322.

[11] M. Campillo, C. Dascalu and I. R. Ionescu, Instability of a Periodic System of Faults, Geophysical Journal, International, vol. 159, 2004, pp. 212-222.

[12] G.A. Chechkin and R.R. Gadyl'shin, A boundary value problem for the Laplacian with Rapidly Changing Type of Boundary Conditions in a Multidimensional Domain, Siberian Mathematical Journal, vol. 40, 2, 1999, pp. 229-244. MR1698303 (2000d:35011)

[13] F. Cotton and M. Campillo, Frequency domain inversion of strong motions: application to the 1992 earthquake, J. Geophys. Res., vol. 100, 1995, pp. 3961-3975.

[14] D. Cioranescu and F. Murat, Un terme etrange venu d'ailleurs, Nonlinear partial differential equations and their applications, College de France Seminar, II \& III, H. Brezis and J.L. Lions, eds., Research Notes in Math. no. 60870, Pitman, 1982, pp. 98-138, 154-178. MR0652509 (84e:35039a) 
[15] A. Damlamian, Le probleme de la passoire de Neumann, Rend. Sem. Mat. Univ. Politecn. Torino, 43, 3, 1985, pp. 427-450. MR0884870 (89c:35014)

[16] C. Dascalu, I.R. Ionescu and M. Campillo, Fault finiteness and initiation of dynamic shear instability, Earth and Planetary Science Letters, vol. 177, 2000, pp. 163-176.

[17] C. Dascalu and I. R. Ionescu, Slip weakening friction instabilities: eigenvalue analysis, Math. Mod. and Methods in Appl. Sci. (M3AS), vol. 3 (14), 2004, no. 3, 439-459. MR2047579

[18] J.H. Dieterich, A model for the nucleation of earthquake slip, Earthquake source mechanics, Geophys. Monogr. Ser., vol. 37, S. Das, J. Boatwright, and C.H. Scholz, eds., AGU, Washington, D.C. (1986), pp. 37-47.

[19] W.L. Elsworth and G.C. Beroza, Seismic evidence for an earthquake nucleation phase, Science, vol. 268, 1995, pp. 851-855.

[20] P. Favreau, M. Campillo and I. R. Ionescu, Initiation of Instability under Slip Dependent Friction in Three Dimension, Journal of Geophysical Research, vol. 107 (B7), 2002.

[21] Y. Iio, Slow initial phase of the P-wave velocity pulse generated by microearthquakes, Geophys. Res. Lett., vol. 19 (5), 1992, pp. 477-480.

[22] I.R. Ionescu, Viscosity solutions for dynamic problems with slip-rate dependent friction, Quart. Appl. Math., vol. LX, No. 3, 2002, 461-476. MR1914436 (2003d:35253)

[23] I.R. Ionescu and M. Campillo, Numerical Study of Initiation: Influence of Non-Linearity and Fault Finiteness, J. Geophys. Res., vol. 104, 1999, pp. 3013-3024.

[24] I.R. Ionescu and J-C. Paumier, On the contact problem with slip dependent friction in elastostatics, Int. J. Eng. Sci., vol. 34(4), 1996, pp. 471-491. MR1383746 (97g:73090)

[25] M. Lobo and E. Perez, Asymptotic behavior of an elastic body with a surface having small sticked regions, Math. Modelling and Num. Analysis, vol. 22, 4, 1988, pp. 609-624. MR0974290 (90b:73053)

[26] R. Madariaga, K. Olsen and R. Archuleta, Modeling dynamic rupture in a 3D earthquake model, Bull. Seism. Soc. Am., vol. 88, 1998, pp. 1182-1197.

[27] D. Onofrei and B. Vernescu, Asymptotic analysis of a Steklov type problem associated to the Newmann sieve model, Anal. App., to appear.

[28] G. Nguetseng and E. Sanchez-Palencia, Stress cocentration for defects distributed near a surface, in Local Effects in the Analysis of Structures, P. Ladevèze, ed., Elsevier, Amsterdam, 1985. MR:0837010(87h:73019)

[29] M. Ohnaka, Y. Kuwahara and K. Yamamoto, Constitutive relations between dynamic physical parameters near a tip of the propagation slip during stick-slip shear failure, Tectonophysics, vol. 144, 1987, pp. 109-125.

[30] A.S. Papageorgiou and K. Aki, A specific barrier model for the quantitative description of inhomogeneous faulting and the prediction of strong ground motion Part I. Description of the model, Bull. Seism. Soc. Am., vol. 73, 1983, pp. 693-722.

[31] A.S Papageorgiou and K. Aki, A specific barrier model for the quantitative description of inhomogeneous faulting and the prediction of strong ground motion Part II. Applications of the model, Bull. Seism. Soc. Am., vol. 73, 1983, pp. 953-978.

[32] H. Perfettini, M. Campillo and I. R. Ionescu, Rescaling of the weakening rate, Geophysical Journal Letters, vol. 108, B9, 2003, pp. 2410.

[33] C.H. Scholz, The Mechanics of Earthquakes and Faulting, Cambridge University Press, Cambridge, 1990.

[34] K. Uenishi and J. Rice, Universal nucleation length for slip-weakening rupture instability under non-uniform fault loading, J. Geophys. Res., 108(B1), cn:2042, doi:10.1029/2001JB001681, 2003, pp. ESE 17-1 17-14.

[35] C. Voisin, I. R. Ionescu and M. Campillo, Crack growth resistance and dynamic rupture arest under slip dependent friction, Physics of the Earth and Planetary Interiors, vol. 131, 2002, pp. 279-294. 